\title{
新型无机二维材料在气体分离膜领域的研究进展
}

\author{
杨浏金金 ${ }^{1,2}$, 罗文华 ${ }^{2}$, 汪长安 ${ }^{1}$, 徐 晨 ${ }^{2}$ \\ (1. 清华大学 材料科学与工程学院, 北京 100084; 2. 中国工程物理研究院 材料研究所, 江油 621700)
}

摘 要: 气体膜分离技术是过滤与分离工业的重要技术之一, 相比于传统分离技术更加高效、节能、环保。新型无机 二维材料在分离膜领域的应用，有望同时实现高选择性和高渗透率，突破商业聚合物膜渗透率和选择性相互制约的 瓶颈，极大地促进高性能分离膜的发展。本文简述了膜的气体分离机制，综述了石墨烯基、过渡金属硫族化物(TMDs) 和二维过渡金属碳化物氮化物(MXene)等新型无机二维材料近年来在气体分离膜领域的研究进展, 包括其设计、制造 和应用, 探讨了不同材料分离膜的特点、面临的挑战和发展前景。此外, 本文对其他新兴二维材料一一层状双氢氧化 物( $\mathrm{LDHs}) 、$ 六方氮化嗍(h-BN)、云母纳米片等的分离膜研究也进行了概述。最后, 对新型无机二维材料在气体分离膜 领域的研究方向及面临的挑战作出了评价。

关 键 词: 膜; 气体分离; 无机材料; 二维材料; 综述

中图分类号: TQ174 文献标识码: A

\section{Novel Inorganic Two-dimensional Materials for Gas Separation Membranes}

\author{
YANG Liuxin $^{1,2}$, LUO Wenhua ${ }^{2}$, WANG Changan ${ }^{1}$, XU Chen ${ }^{2}$ \\ (1. School of Materials Science and Engineering, Tsinghua University, Beijing 100084, China; 2. Institute of Materials, China \\ Academy of Engineering Physics, Jiangyou 621700, China)
}

\begin{abstract}
Membrane-based gas separation is one of the critical technologies in filtration and separation industry, since it is more efficient, energy-saving and environmentally friendly compared with traditional separation technologies. Novel inorganic two-dimensional materials (2DMs) for gas separation are expected to achieve both high selectivity and high permeability, breaking through the trade-off between selectivity and permeability of commercial polymer membranes. This review begins with a brief explanation of gas separation mechanisms for membranes. Afterwards, special attention will be given to the recent advances in novel inorganic 2DMs including graphene and their derivatives, TMDs and MXene, about their design, fabrication and application in gas separation. The gas separation characteristics of different materials, their challenges and directions for future research are summarized. Moreover, the application of other novel inorganic 2DMs, such as LDH, h-BN and mica nanosheets in gas separation technology is also discussed. Finally, the perspectives and challenges for future research of novel inorganic 2DMs in gas separation field are outlined.
\end{abstract}

Key words: membrane; gas separation; inorganic material; two-dimensional material; review

自 1979 年“Prism”气体分离膜装置实现商业化 应用以来，膜分离迅速发展成为一种具有竞争力的
分离技术，是解决能源短缺、环境污染等全球性问 题的重要途径之一 ${ }^{[1-2]}$ 。气体膜分离是一种压力驱动

收稿日期: 2019-10-28; 收到修改稿日期：2019-12-04

基金项目: 中国工程物理研究院院长基金(YZJJLX2017009); 中国工程物理研究院材料研究所特聘基金(TP20160208); 中 国工程物理研究院材料研究所统筹授权项目(TCSQ2016213)

The Dean Foundation of China Academy of Engineering Physics (YZJJLX2017009); Foundation of Institute of Materials, China Academy of Engineering Physics (TP20160208); Project of Institute of Materials, China Academy of Engineering Physics (TCSQ2016213)

作者简介: 杨汶釒金(1995-), 女，博士研究生. E-mail: yanglx13@foxmail YANG Liuxin(1995-), female, PhD candidate. E-mail: yanglx13@foxmail

通讯作者：徐 晨，副研究员. E-mail: chenxuacademic@163.com

XU Chen, associate professor. E-mail: chenxuacademic@163.com 
过程, 广泛应用于天然气的分离净化、空气中的富 氧与浓氮、有机气体分离、工业气体净化等领域 ${ }^{[3-6]}$ 。 与传统分离方法(如低温精馏法和变压吸附法)相比, 气体膜分离过程不发生相变, 具有节约能源、对环 境友好、可实现连续分离、降低生产成本、缩小设 备尺寸等优点 ${ }^{[1,7-8]}$ 。

目前, 在工业领域广泛应用的气体分离膜材料 是传统有机高分子材料, 主要包括纤维素类、聚酰 胺类、聚砜类、有机硅聚合物类等 ${ }^{[9]}$ 。然而, 商业化 聚合物膜存在渗透率和选择性相互制约(Trade-off) 的问题, 减小膜的厚度可以提高气体渗透率但会降 低选择性 ${ }^{[10-11]}$ 。无机二维(2D)材料膜的出现打破了 Trade-off 效应, 因为无机 2D 材料膜具有超薄的厚度, 可以极大地提高渗透率, 同时, 其气体分离机制与 聚合物膜不同, 在超薄的厚度下, 仍能保持高选择 性 ${ }^{[12-14]}$ 。此外, 无机 $2 \mathrm{D}$ 材料膜通常具有耐高温和耐 化学腐蚀的优点, 为长期使用提供了可靠的保障。

目前, 研究最为广泛的无机 $2 \mathrm{D}$ 材料膜是石墨 烯基膜, 包括多孔石墨烯膜和氧化石墨烯膜等。其 他新兴无机 2D 材料, 如过渡金属硫族化物、二维过 渡金属碳化物/氮化物等在气体分离膜领域的应用, 近年来也引起了研究人员的关注。本文综述了新型 无机 $2 \mathrm{D}$ 材料在气体分离膜领域的研究进展, 并指 出其面临的挑战和未来发展的方向。

\section{1 膜的气体分离机制}

传统分离膜中, 非多孔膜(如致密的聚合物膜) 通过溶解-扩散机制传质, 分子由于溶解或扩散能
力不同而分离 ${ }^{[10,15]}$ (图 1(a)); 多孔膜的分离机制由 孔径 $\left(D_{\mathrm{p}}\right)$ 、分子直径 $\left(D_{\mathrm{m}}\right)$ 、分子平均自由程 $(\lambda)$ 等因 素决定(图 1(b, c)): 若 $D_{\mathrm{p}}$ 允许小分子通过, 静态阻 碍大分子通过, 称为分子篮效应; 若 $D_{\mathrm{p}}$ 远远大于 $D_{\mathrm{m}}$, 但小于 $\lambda$, 传质由克努森扩散控制, 分子量小 的气体扩散快 ${ }^{[11,14]}$ 。此外, 还有表面扩散、毛细凝 聚等多种扩散机制 ${ }^{[14]}$ 。

无机 $2 \mathrm{D}$ 材料膜包括纳米多孔原子级薄膜 (Nanoporous Atomic Thin Film, NATM)、2D 材料层 状膜和混合基质膜。NATM 是指具有亚纳米孔的单 层 $2 \mathrm{D}$ 材料膜(图 1(d)), 目前被广泛接受的分离机理 是: 当 $D_{\mathrm{p}}$ 略大于 $D_{\mathrm{m}}$ 而小于 $\lambda$ 时, 传质受空间位阻 限制, 渗透率高而选择性低; 当 $D_{\mathrm{p}}$ 和 $D_{\mathrm{m}}$ 相当或略 小一点时, 分子进入孔需要克服很大的能垒, 分子 键可能发生拉伸或弯曲, 称为活化机制, 此时膜具 有高选择性和低渗透率; 若 $D_{\mathrm{p}}$ 介于两种分子的 $D_{\mathrm{m}}$ 之间，小分子受空间位阻限制，大分子受活化机制 控制, 此时膜兼具高选择性和高渗透率, 是理想的 孔径 ${ }^{[14]}$ 。此外, 纳米孔边缘的表面电荷或官能团也 可能影响分子的渗透 ${ }^{[18]}$ 。

2D 材料层状膜对气体分子的主要传质路径为 (图 1(e)): 层与层之间的二维通道 ${ }^{[19]}$ 、片层之间的缝 隙以及片层内部的结构缺陷 ${ }^{[20]}$ 。足够小的层间距可 以通过分子篮或克努森效应实现气体分离, 而片层 内部的缺陷可能通过与 NATM 相似的机制分离气 体 ${ }^{[1,19-20]}$ 。二维材料表面的官能团与气体分子的相互 作用也会影响其渗透, 如氧化石墨烯膜上的 $-\mathrm{COOH}$ 和 $-\mathrm{OH}$ 与极性 $\mathrm{C}=\mathrm{O}$ 键相互作用, 有利于 $\mathrm{CO}_{2}$ 的吸附 ${ }^{[19-20]}$ 。

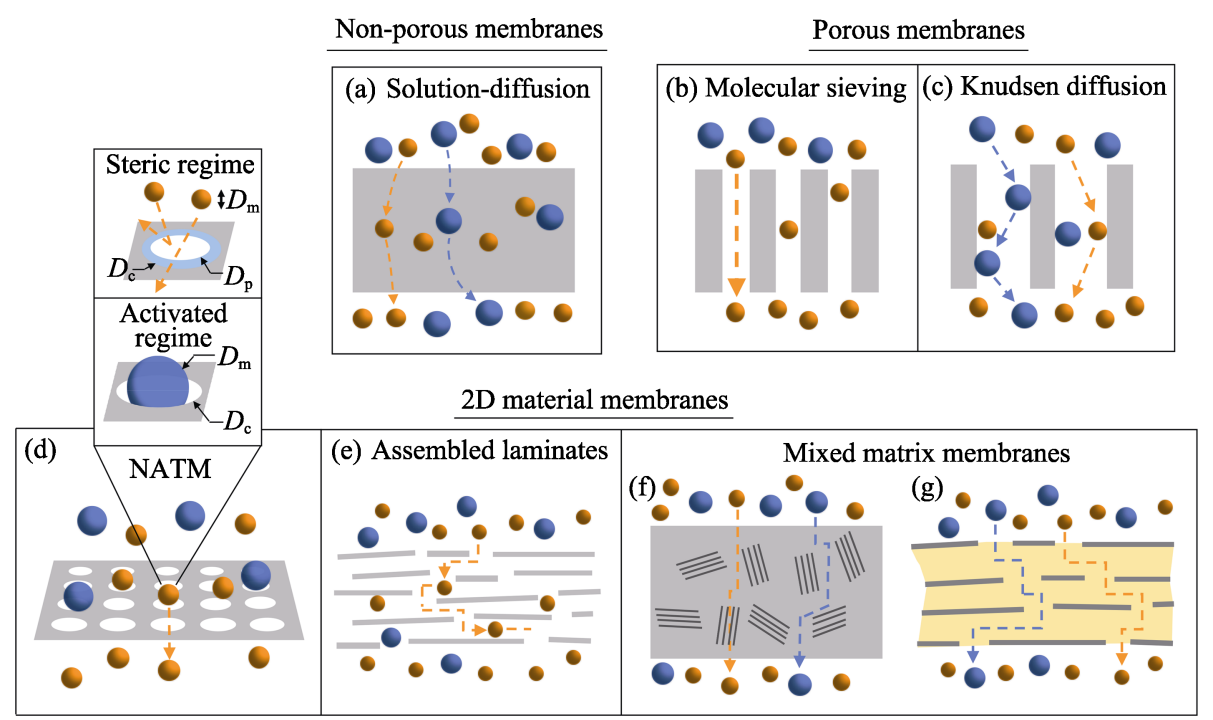

图 1 膜的传输机制示意图, 孔直径定义为 $D_{\mathrm{p}}=D_{\mathrm{c}}-D_{\mathrm{vdw}} / \sqrt{2}$, 其中 $D_{\mathrm{c}}$ 是碳原子中心之间的直径, $D_{\mathrm{vdw}}$ 是碳原子的范德华直径 $[1,10,11,14-17]$

Fig. 1 Schematic representation of transport mechanisms in membranes, where $D_{\mathrm{c}}$ is the location of carbon centers, and the pore diameter is defined as $D_{\mathrm{p}}=D_{\mathrm{c}}-D_{\mathrm{vdw}} / \sqrt{2}$, where $D_{\mathrm{vdw}}$ is the van der Waals diameter of a carbon atom ${ }^{[1,10,11,14-17]}$ 
二维材料混合基质膜的分离机理更为复杂(图 1(f, g))。因为混合基质膜形式多样, 受材料的复合 形式、片层的分散状态、有机基质的化学特性等因 素影响，可能综合溶解-扩散、分子篎效应、克努森 扩散等多种分离机制 ${ }^{[1,21-22]}$ 。

\section{2 石墨烯基分离膜}

石墨烯，是由 $\mathrm{sp}^{2}$ 杂化的碳原子构成的六角型 蜂巢状单层、双层或少层(3 10)2D 石墨晶体 ${ }^{[23-24]}$, 表面较为平整, 可以最小化传质阻力、最大化渗透 率, 同时具有优异的机械强度和化学稳定性, 因此 在膜分离领域有一定的应用前景。氧化石墨烯 $(\mathrm{GO})$ 是石墨烯的氧化衍生物, 具有亲水性, 可以均匀分 散在水溶液中, 通过真空抽滤、旋涂、溶液浇铸等 方法组装成 GO 层状膜。

本文所述石墨烯基膜包括多孔石墨烯膜、氧化 石墨烯层状膜和石墨烯混合基质膜。

\section{1 多孔石墨烯}

单层无缺陷的石墨烯不能渗透气体(包括氦 气 $)^{[25-26]}$ 。然而, 石墨烯超薄的厚度和极高的平面抗 拉强度 ${ }^{[14,27]}$ 使其可以通过 “打孔”成为性能良好的分 离膜——多孔石墨烯膜。多孔石墨烯膜是 NATM 的 一种，主要基于孔的尺寸效应进行气体分离。

计算是对材料的结构和性能进行预测或设计的 重要手段。Jiang 等 ${ }^{[28]}$ 通过第一性原理计算, 论证了 多孔石墨烯应用于气体分离的可能性，同时证明了 修饰孔边缘的原子会影响分离性能: 他们设计的两 种多孔石墨烯分别具有部分氢针化的孔边缘和全部 氢针化的孔边缘, 对 $\mathrm{H}_{2} / \mathrm{CH}_{4}$ 的选择性高达 $10^{8}$ 和 (a)

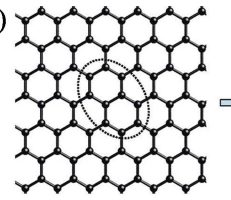

(c)

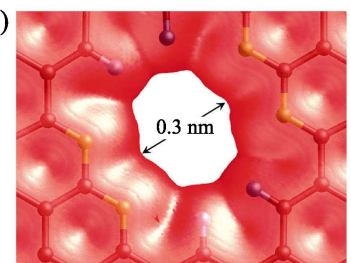

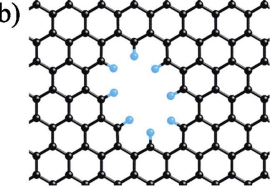

(d)

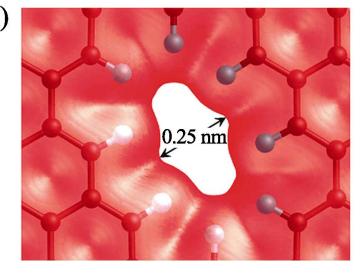

图 2 石墨烯上形成纳米孔的示意图 $(\mathrm{a} \sim \mathrm{b})$ 和多孔石墨烯的 孔电子密度等值面 $(\mathfrak{c} \sim \mathrm{d})^{[28]}$

Fig. 2 Schematic of pores in graphene sheet (a-b) and pore electron density isosurfaces of porous graphene $(\mathrm{c}-\mathrm{d})^{[28]}$

(a) Creation of a nitrogen-functionalized pore within a graphene sheet; (b) An all-hydrogen passivated pore in graphene; (c) Pore electron density isosurface of the nitrogen-functionalized porous graphene; (d) The all-hydrogen passivated porous graphene

Color code: C-black; N-green; H-cyan (isovalue is at $0.2 \mathrm{e} / \mathrm{nm}$ )

$10^{23}$ (图 2)。Wang 等 ${ }^{[29]}$ 通过计算设计了双层多孔石 墨烯, 在单层孔尺寸为 $2.5 \mathrm{~nm}$ 时, 改变两层石墨烯 的相对位置, 可以使孔的有效尺寸达到 $0.36 \mathrm{~nm}$, 从 而实现对 $\mathrm{CO}_{2} 、 \mathrm{~N}_{2}$ 和 $\mathrm{CH}_{4}$ 的有效分离(图 3)。

上述均为模拟计算的结果。实验上, 研究人员 通过紫外线氧化刻蚀 ${ }^{[30]}$ 、离子束钻孔 ${ }^{[28,31-32]}$ 、臭氧 氧化刻蚀 ${ }^{[33]}$ 、电子束辐射 ${ }^{[34-37]}$ 等方法制备出一系列 多孔石墨烯膜。Koenig 等 ${ }^{[30]}$ 利用紫外线诱导氧化刻 蚀法在机械剥离的石墨烯膜上打孔，测量了 $\mathrm{H}_{2}$ 、 $\mathrm{CO}_{2} 、 \mathrm{Ar} 、 \mathrm{~N}_{2}$ 等多种气体通过孔隙的传输特性, 首 次从实验上证明了多孔石墨烯膜的气体篎分功能。 Park 等 ${ }^{[32]}$ 使用 $\mathrm{He}$ 聚焦离子束在化学气相沉积法 (CVD 法)制备的双层石墨烯膜上打孔, 获得的膜具
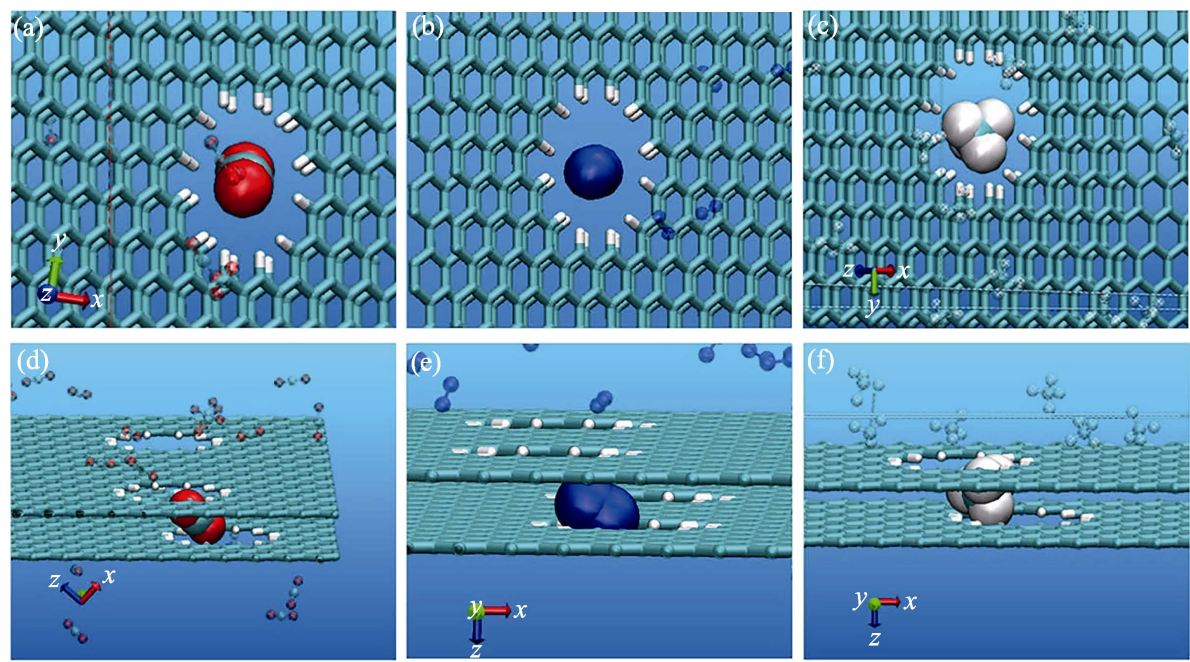

图 3 气体分子通过孔的示意图 ${ }^{[29]}$

Fig. 3 Snapshots of passing-through events of gas molecules ${ }^{[29]}$ (a, d) $\mathrm{CO}_{2}$; (b, e) $\mathrm{N}_{2}$; (c, f) $\mathrm{CH}_{4}$. (a-c) Top view; (d-f) Side view 


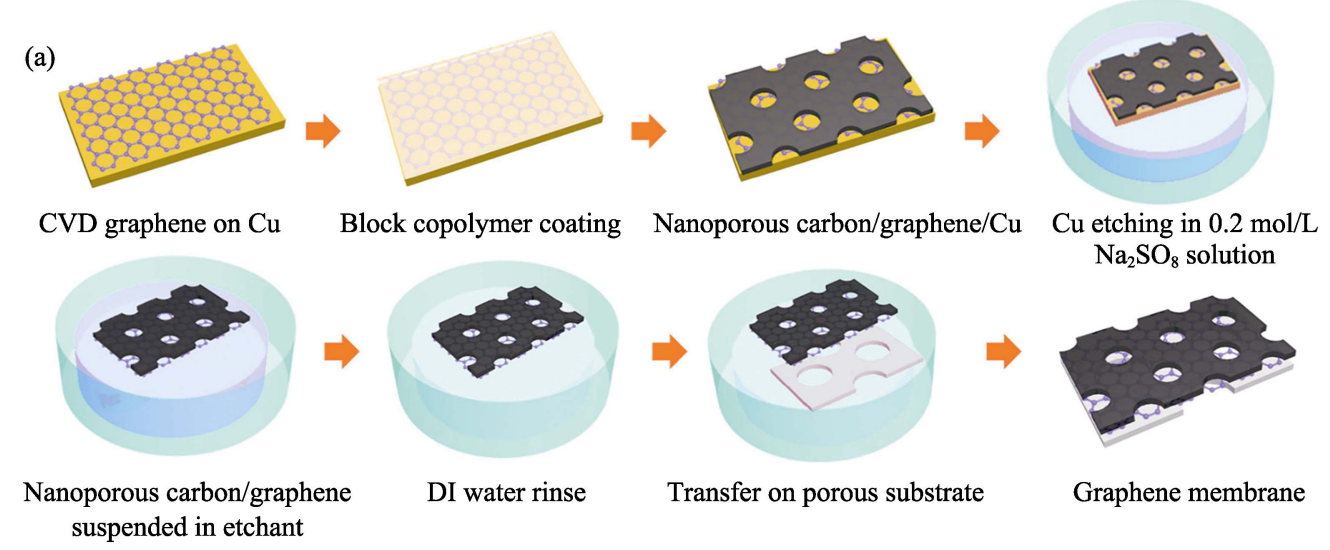

(b)
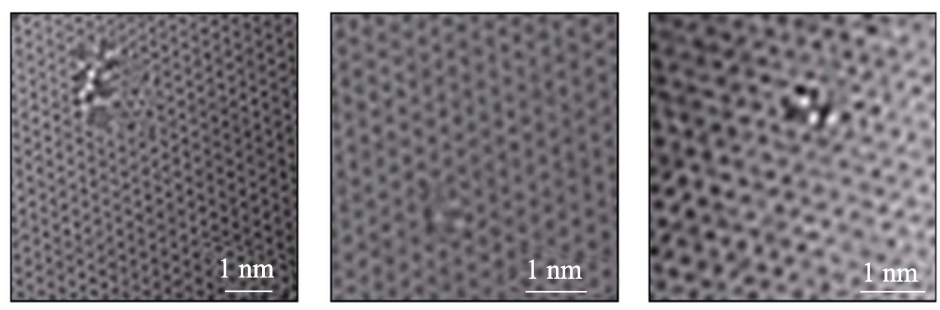

图 4 纳米多孔碳膜辅助转移法制备大面积石墨烯膜示意图(a)和石墨烯点阵中本征缺陷的高分辨 TEM 照片(b) ${ }^{[33]}$

Fig. 4 Schematic of fabrication of a large-area graphene membrane by the nanoporous carbon film-assisted transfer method (a) and high-resolution TEM images of the intrinsic defects in graphene lattice $(b)^{[33]}$

有较高的气体渗透率, 但因孔径较大而选择性较低, 对 $\mathrm{H}_{2} / \mathrm{CO}_{2}$ 的选择性仅为 4.69 。机械剥离法制备的石 墨烯尺寸较小、可控性较低、难以大规模合成。相 比之下, CVD 法是目前制备大面积、高质量石墨烯 最具潜力的方法之一, 但在转移石墨烯时可能引入 缺陷和裂痕, 影响膜的选择性和机械强度。因此, 无 缺陷地转移石墨烯是 CVD 法的一大难点。Kumar 等 ${ }^{[33]}$ 利用纳米多孔碳膜辅助转移法, 制备出大面积 $\left(1 \mathrm{~mm}^{2}\right)$ 、无缺陷的单层石墨烯膜, 同时在 CVD 过 程中引入臭氧刻蚀, 获得的多孔石墨烯膜具有较高 的 $\mathrm{H}_{2} / \mathrm{CH}_{4}$ 选择性(25)和高 $\mathrm{H}_{2}$ 渗透率(图 4)。

多孔石墨烯膜的特点是厚度非常薄, 传质路径 短, 通过孔的尺寸效应分离气体, 渗透率取决于孔 的数量, 理论选择性非常高。然而, 由于制孔技术尚 不成熟, 实验选择性与理论值差距较大。因此, 优化 和开发更加先进的制孔技术，制备孔径理想均一、 高密度、均匀分布的亚纳米孔，是提高多孔石墨烯 膜实际性能的关键。

\section{2 氧化石墨烯}

对于多孔石墨烯膜，大面积、高质量石墨烯的规 模化制备及精确制孔仍是巨大的挑战。相比之下, GO 纳米片可以通过改良的 Hummers 法大量生产 ${ }^{[38-39]}$, 且更容易组装成层状膜。此外, GO 的含氧基团也有 利于对其进行功能化修饰。2012 年, Nair 等 ${ }^{[40]}$ 发现 亚微米厚度的 GO 层状膜不能透过包括氦气在内的 多种气体，但可以迅速透过水分子(图 5)。这一独特
的传质特性引发了 $\mathrm{GO}$ 膜在分离和过滤领域的研究 热潮。

随后的研究发现纳米级厚度的 GO 膜可以选择 性透过气体分子 ${ }^{[19-20]}$, 具有优异的气体分离性能, 这与它的二维通道微结构、层间距、本征缺陷等有 关。Park 等 ${ }^{[19]}$ 比较了两种方法制备的 GO 层状膜，它 们的微结构不同, 有序紧密堆积的 GO 膜具有更曲 折的气流通道, 可以实现高 $\mathrm{CO}_{2} / \mathrm{N}_{2}$ 选择性(图 6)。 $\mathrm{GO}$ 的平面尺寸也会影响通道微结构, 从而影响膜 分离的效率。大尺寸 $\mathrm{GO}$ 组装的层状膜对 $\mathrm{H}_{2} / \mathrm{CO}_{2}$ 的选择性优于小尺寸 $\mathrm{GO}$ 组装的膜, 原因是大尺寸 GO 片层间的二维通道更曲折，气体分子的传质路 径更长 ${ }^{[41]}$ 。

层间距 ( $d$-spacing) 是二维通道的重要参数, 对 分离性能具有显著影响, 引入聚合物可有效调节层 间距。Sun 等 ${ }^{[22]}$ 利用硫脲共价交联 GO 膜, 将 GO 膜的层间距从 $0.84 \mathrm{~nm}$ 减小到 $0.71 \mathrm{~nm}$, 从而提高氢

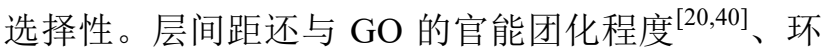
境湿度 ${ }^{[40,43]}$ 以及 $\mathrm{GO}$ 的组装方式 ${ }^{[19,44]}$ 等因素有关。 $\mathrm{Kim}$ 等 ${ }^{[45}$ 将 $\mathrm{GO}$ 膜在 $\mathrm{HI}$ 蒸汽中暴露 $0 \sim 5 \mathrm{~min}$, 实现 了对 $\mathrm{GO}$ 不同程度的还原, 层间距为 $1.15 \sim 0.37 \mathrm{~nm}$ 。 然而, 还原程度过高的 $\mathrm{GO}$ 膜 $(d \sim 0.36 \mathrm{~nm})$, 与石墨 层间距相近, 无法渗透气体 ${ }^{[46]}$ 。

$\mathrm{GO}$ 片层内的本征缺陷也是气体分子的传质路 径之一。Yu 等 ${ }^{[20]}$ 通过简单的真空抽滤法制备出 $1.8 \mathrm{~nm}$ 的超薄 $\mathrm{GO}$ 膜, 对 $\mathrm{H}_{2} / \mathrm{CO}_{2}$ 和 $\mathrm{H}_{2} / \mathrm{N}_{2}$ 的选择性 

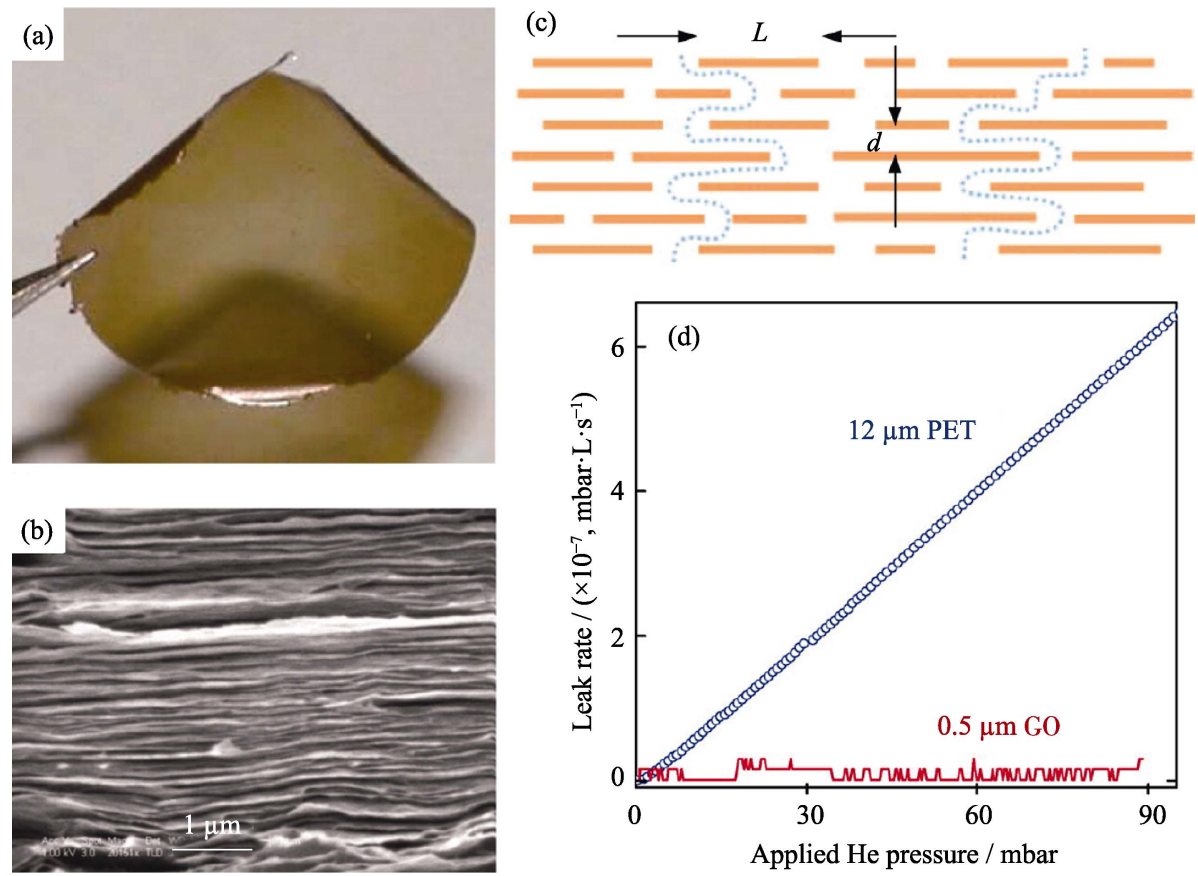

图 $51 \mu \mathrm{m}$ 厚 GO 膜的照片(a), 分离膜的截面 SEM 照片(b)，分子在 GO 膜层间的渗透路径示意照片(c)和

自支撑的亚微米厚 GO 膜和对照组 PET 膜的 He 渗透性能对比 $(\mathrm{d})^{[40]}(1 \mathrm{mbar}=100 \mathrm{~Pa})$

Fig. 5 Photo of a 1- $\mu$ m-thick GO film (a), electron micrograph of the film's cross section (b), schematic view for possible permeation through the laminates (c), and examples of He-leak measurements for a freestanding submicrometer-thick GO membrane and a reference PET film $(\mathrm{d})^{[40]}(1 \mathrm{mbar}=100 \mathrm{~Pa})$

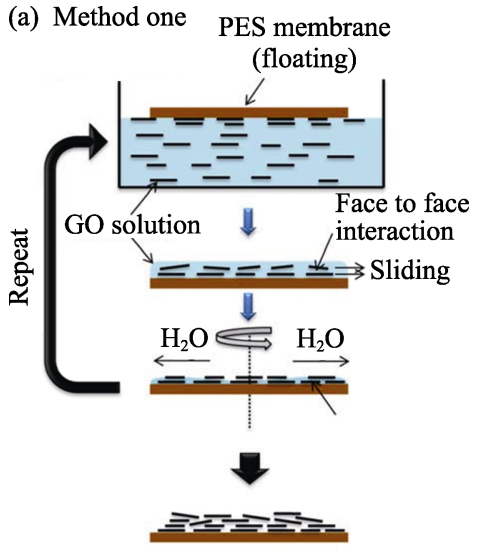

Less interlocked

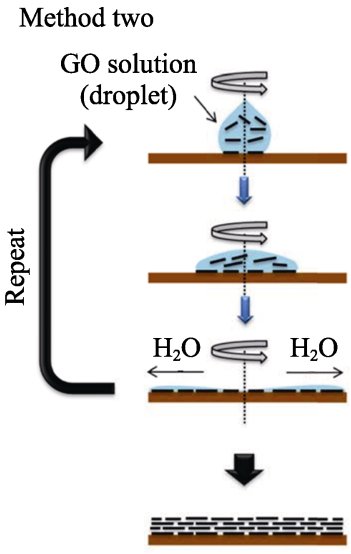

Highly interlocked (b)
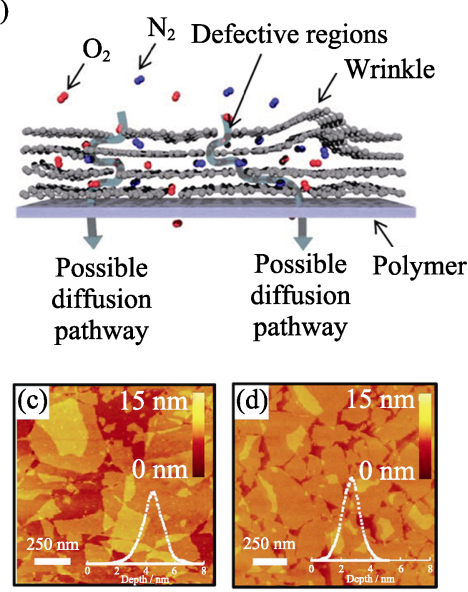

图 6 不同的 GO 膜制备方法示意图(a), GO 膜可能的气体传输途径示意图(b), GO 膜表面的 AFM 图与表面深度分布(c,d) ${ }^{[19]}$

Fig. 6 Schematic illustration of two different GO coating methods (Method one and Method two) (a), schematic illustration of possible gas transport through graphene membrane (b), AFM images of GO membrane surfaces with insert images showing depth profiles of GO membrane surfaces: (c) method one [root mean square roughness $\left(R_{\mathrm{q}}\right)=0.8 \mathrm{~nm}$, average roughness $\left.\left(R_{\mathrm{a}}\right)=0.614 \mathrm{~nm}\right]$ and (d) method two $\left(R_{\mathrm{q}}=0.608 \mathrm{~nm}, R_{\mathrm{a}}=0.467 \mathrm{~nm}\right)^{[19]}$

分别为 3400 和 900 , 是目前报道的最高的选择系 数。GO 膜经还原后层间距变小, 但气体渗透率没有 明显改变, 这说明气体分子主要的传质路径可能是 片层内部的结构缺陷而不是层间通道。

目前，多孔石墨烯膜的制备难度仍较大，实验 选择性远低于理论选择性, 而 GO 层状膜相对更容 易制备出具有选择性的层间通道，普遍具有比多孔 石墨烯膜更高的气体选择性，但不同实验方法制备
的膜的气体渗透率的差异较大。

\section{3 石墨烯混合基质膜}

GO 膜的可加工性不如聚合物膜, 且机械强度需 要进一步提高以适应高压、复杂的实际应用环境 ${ }^{[7]}$ 。 通过与有机物或离子液体复合，开发混合基质膜以 提高膜的可加工性、机械性能和可靠性 ${ }^{[39-41]}$, 是近 年气体分离膜领域的研究热点。复合形式包括将 GO 片层分散于聚合物基体中、将有机物或离子液 

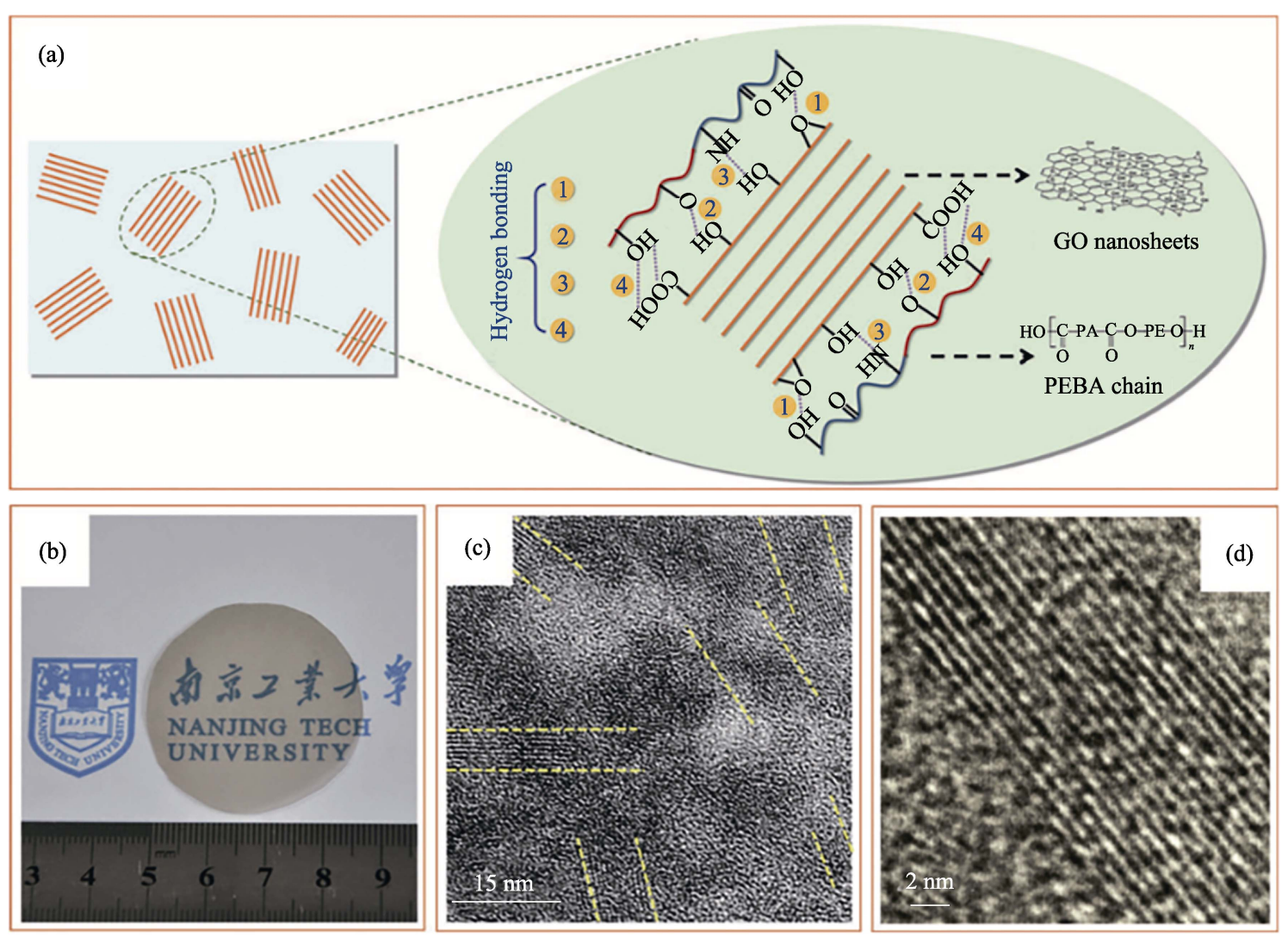

图 7 PEBA 基膜中组装的 GO 纳米片示意图(a), 含有 $0.1 \mathrm{wt} \% \mathrm{GO}$ 的膜(b)和 TEM 照片(黄色虚线指示 GO 片层) (c), 以及 GO-1 膜横截面的 TEM 照片 $(\mathrm{d})^{[17]}$

Fig. 7 Schematic representation of the assembly of GO nanosheets in polymeric environment (a), digital photographs of the membrane with $0.1 \mathrm{wt} \% \mathrm{GO}(\mathrm{b})$, overview (the yellow dashed lines are eye-guiding lines indicating the GO laminates in these regions) (c) and expanded TEM image of the cross section of GO-1 membrane (d ${ }^{[17]}$

体填充于 GO 膜层间等 ${ }^{[48-50]}$ 。

Jin 等 ${ }^{[17]}$ 在聚醚嵌段酰胺(PEBA) 基膜中加入 $0.1 \mathrm{wt} \%$ 的 $\mathrm{GO}$ (图 7), 少层堆叠的 $\mathrm{GO}$ 具有分子篮作 用的层间距 $(0.7 \mathrm{~nm})$ 和较为直接的扩散路径, 获得 的 PEBA-GO 膜具有优异的分离性能。增加 GO 与 聚合物基体的相容性, 减少界面缺陷, 是提高混合 基质膜性能的重要途径 ${ }^{[1-52]}$ 。Xin 等 ${ }^{[53]}$ 用亲 $\mathrm{CO}_{2}$ 的 磺化聚合物分子刷修饰 $\mathrm{GO}(\mathrm{S}-\mathrm{GO})$, 再将其引入磺 化聚醚醚酮(SPEEK)基体中。磺化聚合物可以增强 $\mathrm{GO}$ 与 SPEEK 的相容性, 同时提供亲 $\mathrm{CO}_{2}$ 的位点, 提高膜对 $\mathrm{CO}_{2}$ 的吸附选择性, 而 $\mathrm{GO}$ 有利于提高膜 对 $\mathrm{CO}_{2}$ 的扩散选择性，由此获得的混合基质膜比 SPEEK 对照组对 $\mathrm{CO}_{2} / \mathrm{CH}_{4}$ 的选择性提高 $179 \%$ 。

混合基质膜的另一种形式是在 GO 膜层间填充 另一组分。Jin 等 ${ }^{[54}$ 利用真空旋涂法逐层组装 GO 和 聚乙烯亚胺(PEI), 在机械外力和分子间作用力的协 同作用下, GO 层状膜的微结构变得更为有序、层间 距更小, 具有比商业膜高 2 4 个数量级的 $\mathrm{H}_{2}$ 渗透率 和 3 倍的 $\mathrm{H}_{2} / \mathrm{CO}_{2}$ 选择性(图 8)。Peng 等 ${ }^{[55]}$ 将 $[\mathrm{BMIM}]\left[\mathrm{BF}_{4}\right]$ 离子液体(IL)限制在 $\mathrm{GO}$ 膜层间, 构筑 出 GO 基离子液体复合膜(GO-SILMs) (图 9)。
GO-SILMs 主要通过 IL 层的溶解一扩散机制分离气 体, 阳离子 $[\mathrm{BMIM}]^{+}$与 $\mathrm{GO}$ 键合后, 阴离子 $\left[\mathrm{BF}_{4}\right]^{-}$更 易与 $\mathrm{CO}_{2}$ 键合，使 $\mathrm{CO}_{2}$ 在 IL 层的溶解度增大，从而 提高对 $\mathrm{CO}_{2}$ 的选择性。此外, IL 具有优异的化学稳 定性、热稳定性和高粘度等特点, 使复合膜具有高 机械强度和耐高温高压的特性。

混合基质膜通常具有比聚合物基体更高的气体 选择性和渗透率, 然而与氧化石墨烯膜的最优值相 比仍有一定差距, 但膜的可加工性、机械性能和可 靠性有所提升。表 1 总结了石墨烯基材料在气体分 离膜领域的研究进展。

\section{3 二维过渡金属硫族化物}

石墨烯基膜在气体分离领域的应用，启发了研 究人员对其他无机二维材料的气体分离特性进行探 索。二维过渡金属硫族化物(TMDs) 是一类典型的类 石墨烯材料, 可以通过机械剥离、化学插层剥离、 液相超声剥离等方法 ${ }^{[56-61]}$ 制备, 其中 $\mathrm{MoS}_{2}$ 是研究 最为广泛的 TMDs。Wang 等 ${ }^{[62]}$ 首次将 $\mathrm{MoS}_{2}$ 层状膜 用于气体分离, 他们通过抽滤制得厚度为 $17 \mathrm{~nm}$ 的 

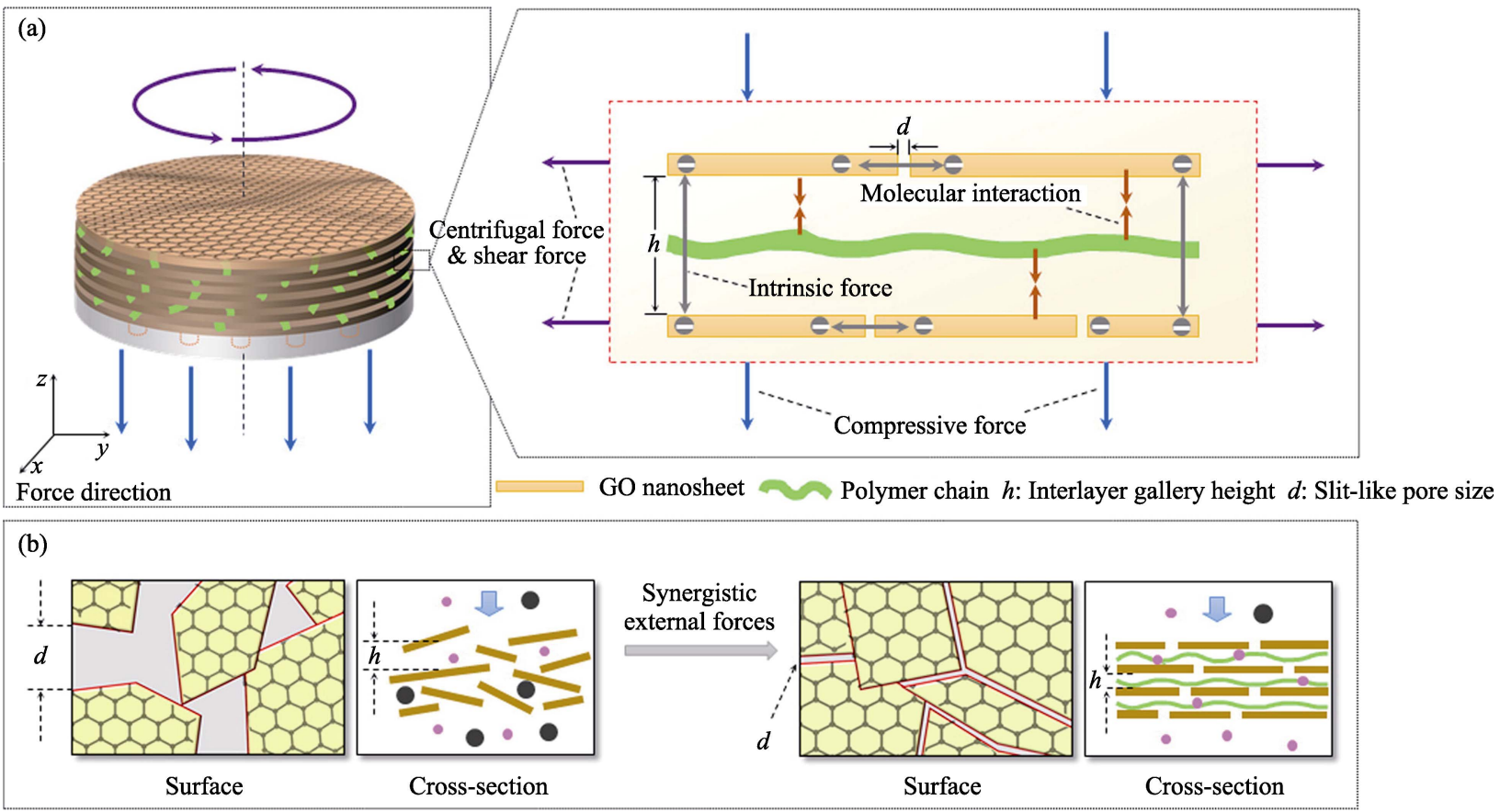

图 $8 \mathrm{GO}$ 纳米片和聚合物链组成的二维通道的受力分析示意图(a), 本征力诱导的无序结构(左)和 协同外力驱动的高度有序层状结构(右)示意图(b) ${ }^{[54]}$

Fig. 8 Force analysis for one 2D channel unit consisting of GO nanosheets and polymer chain (a), schematic illustration of intrinsic force induced disordered structure (left) and highly ordered laminar structures (right) driven by introduced synergistic external forces $(b)^{[54]}$

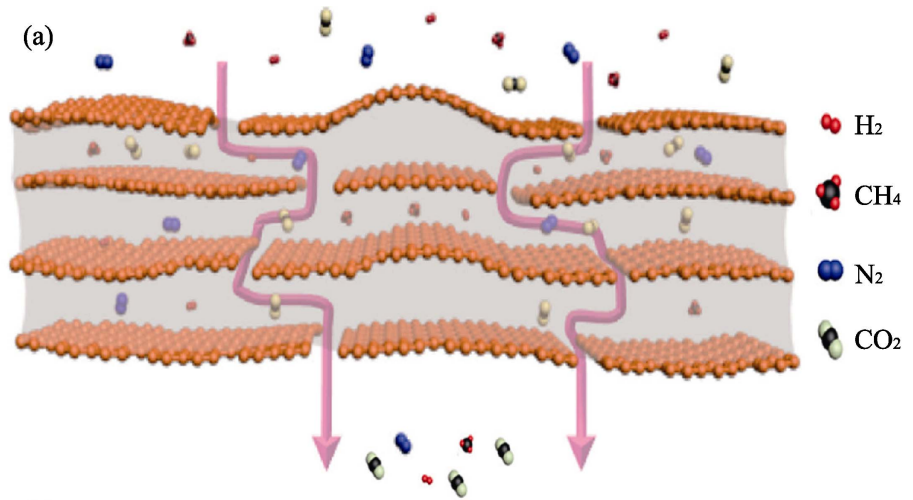

(b)
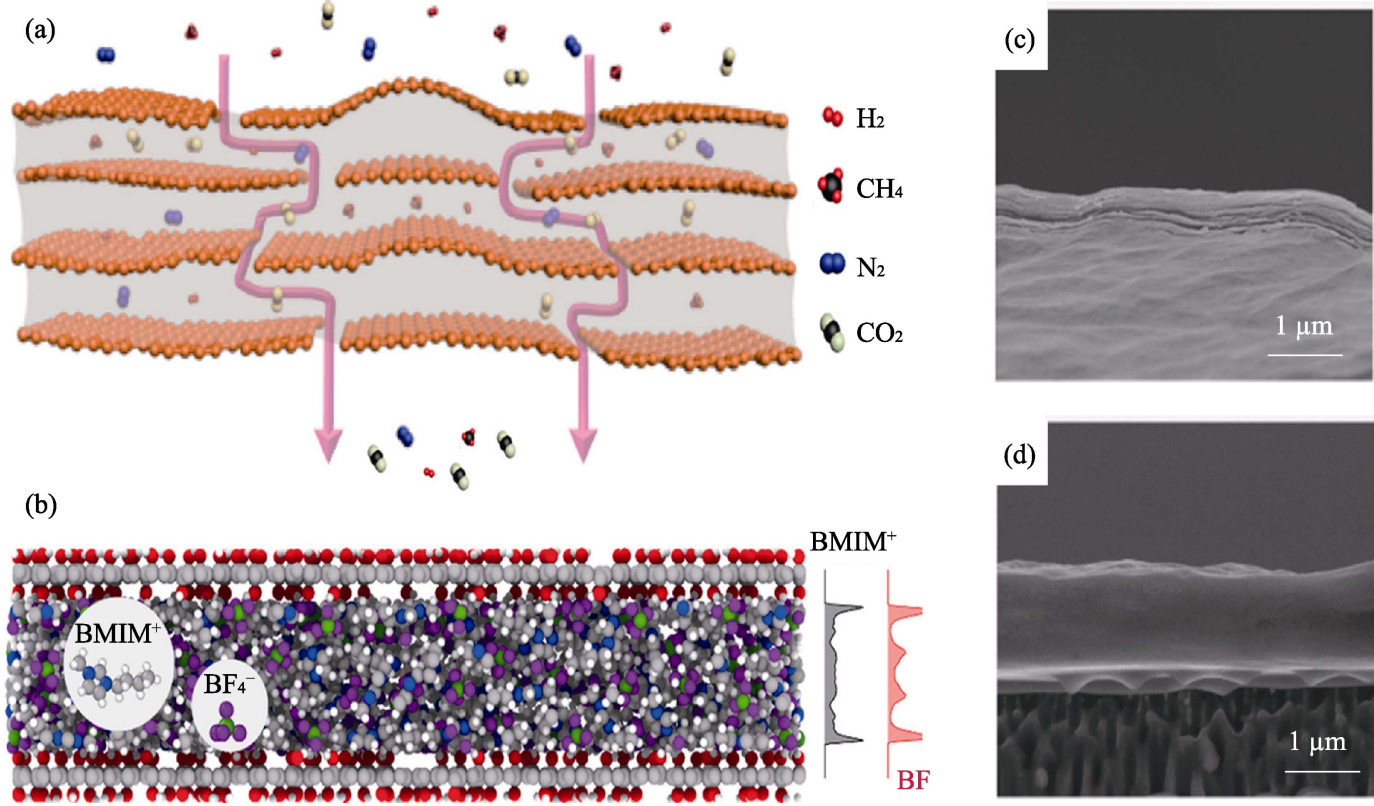

(d)

图 9 气体通过 GO-SILM 膜溶解-扩散传输路径示意图(a), 限制在 GO 纳米通道中的 $[\mathrm{BMIM}]\left[\mathrm{BF}_{4}\right]$ 离子液体分子结构(b), GO 膜(c)和 GO-SILM 膜(d)的截面 SEM 照片 ${ }^{[55]}$

Fig. 9 Schematic illustration of the gas solution-diffusion transport pathway through a GO-SILM (a), molecular structures of $[\mathrm{BMIM}]\left[\mathrm{BF}_{4}\right]$ IL confined in the GO nanochannels $(\mathrm{b})$, the cross section of the GO membrane (c) and GO-SILM (d), respectively ${ }^{[55]}$

$\mathrm{MoS}_{2}$ 膜, 具有高氢渗透率 $\left(9.19 \times 10^{-6} \mathrm{~mol} \cdot \mathrm{m}^{-2} \cdot \mathrm{s}^{-1} \cdot \mathrm{Pa}^{-1}\right)$, 但对 $\mathrm{H}_{2} / \mathrm{CO}_{2}$ 的选择性( 3)很低, 低于克努森值(4.69)。

Achari 等 ${ }^{[63]}$ 率先制备出对 $\mathrm{H}_{2} / \mathrm{CO}_{2}$ 的选择性超 过克努森值的 $\mathrm{MoS}_{2}$ 膜, 并系统研究温度和相转变
对 $\mathrm{MoS}_{2}$ 膜的分离性能的影响。经过 $160{ }^{\circ} \mathrm{C}$ 热处理 $2 \mathrm{~h}$ 后, 膜的气体渗透率增加 $30 \%$, 而选择性几乎不 变。热处理后的 $\mathrm{MoS}_{2}$ 由 $1 \mathrm{~T}$ 相转变为 $2 \mathrm{H}$ 相, 晶面 间距由 $1.132 \mathrm{~nm}$ 缩小为 $0.612 \mathrm{~nm}$, 但膜的总厚度没 
表 1 石墨烯基膜的气体分离性能比较

Table 1 Graphene-based membranes for gas separation

\begin{tabular}{|c|c|c|c|c|c|}
\hline Materials $^{\mathrm{a}}$ & Preparation method & $\begin{array}{c}\text { Feed } \\
\text { condition }\end{array}$ & Selectivity & $\begin{array}{c}\text { Permeate rate/ } \\
\text { permeance/permeability }\end{array}$ & Ref. \\
\hline $\begin{array}{l}\text { Porous graphene monolay- } \\
\text { er }\end{array}$ & Simulation All-H passivation & $\mathrm{H}_{2} / \mathrm{CH}_{4}$ & $10^{23}$ & $10^{-20} \mathrm{~mol} \cdot \mathrm{s}^{-1} \cdot \mathrm{Pa}^{-1}$ & {$[28]$} \\
\hline Porous graphene bilayer & Ultraviolet-induced oxidative etching & $\mathrm{H}_{2} / \mathrm{CH}_{4}$ & 104 & $4.5 \times 10^{-23} \mathrm{~mol} \cdot \mathrm{s}^{-1} \cdot \mathrm{Pa}^{-1}$ & {$[30]$} \\
\hline Porous graphene bilayer & Focused ion beam perforation & $\mathrm{H}_{2} / \mathrm{CO}_{2}$ & 4.6 & $5.0 \times 10^{3} \mathrm{~mol} \cdot \mathrm{m}^{-2} \cdot \mathrm{s}^{-1} \cdot \mathrm{Pa}^{-1}$ & {$[32]$} \\
\hline Porous graphene & $\begin{array}{l}\text { Ozone functionalization-based } \\
\text { pore-etching }\end{array}$ & $\mathrm{H}_{2} / \mathrm{CH}_{4}$ & 25 & $4.1 \times 10^{-7} \mathrm{~mol} \cdot \mathrm{m}^{-2} \cdot \mathrm{s}^{-1} \cdot \mathrm{Pa}^{-1}$ & {$[33]$} \\
\hline \multirow{2}{*}{ GO/PES } & Spin coating & $\mathrm{H}_{2} / \mathrm{CO}_{2}$ & 30 & $4.02 \times 10^{-17} \mathrm{~mol} \cdot \mathrm{m}^{\prime} \mathrm{m}^{-2} \cdot \mathrm{s}^{-1} \cdot \mathrm{Pa}^{-1}$ & \multirow{2}{*}{19} \\
\hline & Dip and spin coating & $\mathrm{H}_{2} / \mathrm{CO}_{2}$ & 20 & $5.69 \times 10^{-17} \mathrm{~mol} \cdot \mathrm{m} \cdot \mathrm{m}^{-2} \cdot \mathrm{s}^{-1} \cdot \mathrm{Pa}^{-1}$ & \\
\hline $\mathrm{GO} / \mathrm{AAO}$ & Vacuum filtration & $\mathrm{H}_{2} / \mathrm{CO}_{2}$ & 3400 & $10^{-7} \mathrm{~mol} \cdot \mathrm{m}^{-2} \cdot \mathrm{s}^{-1} \cdot \mathrm{Pa}^{-1}$ & {$[20]$} \\
\hline $\mathrm{GO} / \mathrm{AAO}$ & Vacuum filtration & $\mathrm{H}_{2} / \mathrm{CO}_{2}$ & 22.5 & $1.14 \times 10^{-7} \mathrm{~mol} \cdot \mathrm{m}^{-2} \cdot \mathrm{s}^{-1} \cdot \mathrm{Pa}^{-1}$ & {$[41]$} \\
\hline TU-GOF & $\begin{array}{c}\text { Hydrothermal } \\
\text { Self-assembly synthesis }\end{array}$ & $\mathrm{H}_{2} / \mathrm{CO}_{2}$ & 225 & $10^{-7} \mathrm{~mol} \cdot \mathrm{m}^{-2} \cdot \mathrm{s}^{-1} \cdot \mathrm{Pa}^{-1}$ & {$[42]$} \\
\hline $\mathrm{GO} / \mathrm{AAO}$ & Spin coating & $\mathrm{H}_{2} / \mathrm{CO}_{2}$ & 240 & $3.4 \times 10^{-7} \mathrm{~mol} \cdot \mathrm{m}^{-2} \cdot \mathrm{s}^{-1} \cdot \mathrm{Pa}^{-1}$ & {$[44]$} \\
\hline PEBA-GO & Drop casting & $\mathrm{CO}_{2} / \mathrm{N}_{2}$ & 91 & $3.35 \times 10^{-14} \mathrm{~mol} \cdot \mathrm{m} \cdot \mathrm{m}^{-2} \cdot \mathrm{s}^{-1} \cdot \mathrm{Pa}^{-1}$ & {$[17]$} \\
\hline SPEEK/S-GO & Drop casting & $\mathrm{CO}_{2} / \mathrm{CH}_{4}$ & 72.2 & $4.44 \times 10^{-13} \mathrm{~mol} \cdot \mathrm{m}^{\prime} \mathrm{m}^{-2} \cdot \mathrm{s}^{-1} \cdot \mathrm{Pa}^{-1}$ & {$[53]$} \\
\hline \multirow[t]{2}{*}{ EFDA-GO } & Vaccum-spin & $\mathrm{H}_{2} / \mathrm{CO}_{2}$ & $29-33$ & $(2.8-4.0) \times 10^{-13} \mathrm{~mol} \cdot \mathrm{m}^{-\mathrm{m}^{-2}} \cdot \mathrm{s}^{-1} \cdot \mathrm{Pa}^{-1}$ & {$[54]$} \\
\hline & & $\mathrm{CO}_{2} / \mathrm{H}_{2}$ & 24 & & \\
\hline \multirow[t]{2}{*}{$\mathrm{GO}-[\mathrm{BMIM}]\left[\mathrm{BF}_{4}\right]$} & Vacuum filtration & $\mathrm{CO}_{2} / \mathrm{CH}_{4}$ & 234 & $2.29 \times 10^{-8} \mathrm{~mol} \cdot \mathrm{m}^{-2} \cdot \mathrm{s}^{-1} \cdot \mathrm{Pa}^{-1}$ & {$[55]$} \\
\hline & & $\mathrm{CO}_{2} / \mathrm{N}_{2}$ & 382 & & \\
\hline
\end{tabular}

${ }^{a}$ PES:Polyethersulfone; AAO: Anodic Aluminum Oxide; PEBA: Polyether Block Amide; SPEEK: Sulfonated Poly-ether-ether-ketone; EFDA: External Force Driven Assembly; TU-GOF: Thiourea-crosslinked graphene oxide Framework
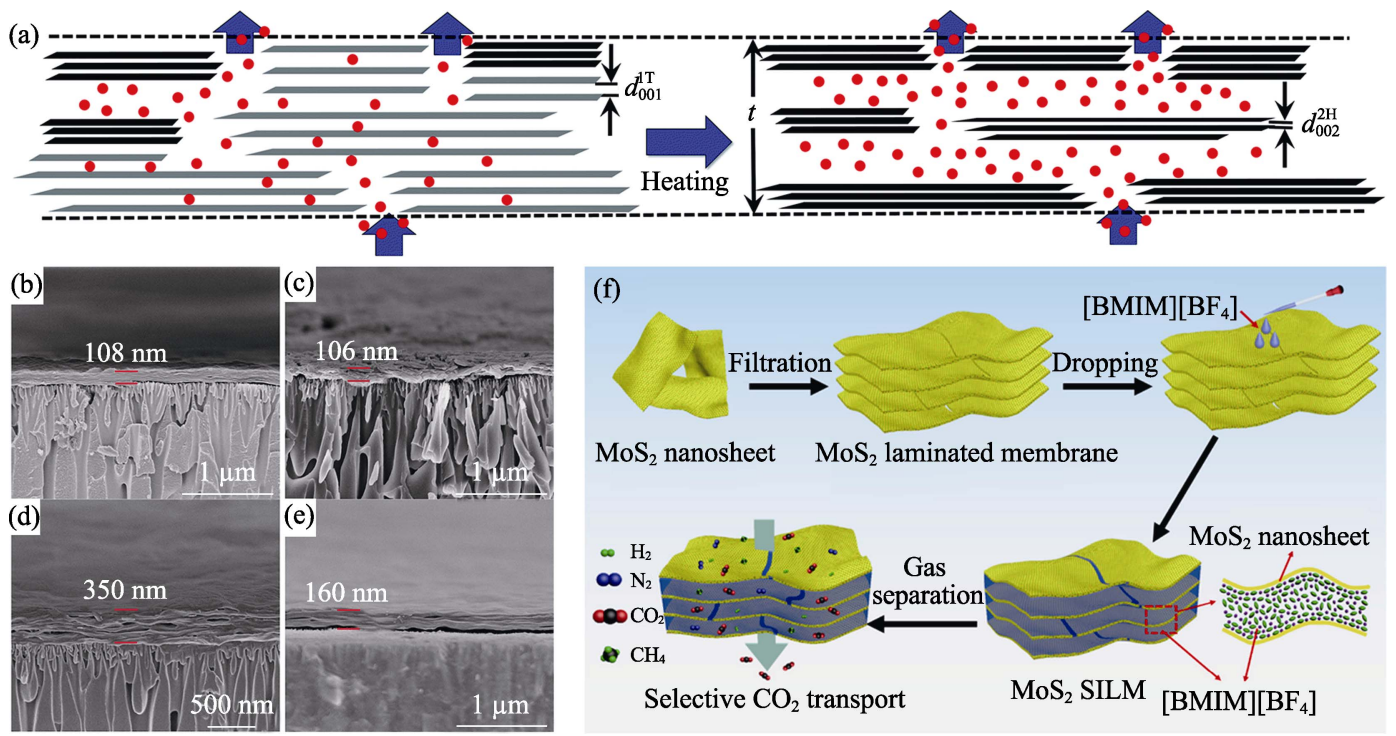

图 $10 \mathrm{MoS}_{2}$ 膜 $160{ }^{\circ} \mathrm{C}$ 热处理前后的气体渗透路径示意图 $(\mathrm{a})^{[63]}$, 膜截面的 $\mathrm{SEM}$ 照片 $(\mathrm{b} \sim \mathrm{e})^{[64]}$ 和 $\mathrm{MoS}_{2}$-SILM 制备过程示意图(f) ${ }^{[69]}$

Fig.10 Schematic of gas permeation pathway across $\mathrm{MoS}_{2}$ membranes before and after heating at $160{ }^{\circ} \mathrm{C}(\mathrm{a})^{[63]}$, cross section SEM images of the membrane (b-e $)^{[64]}$, and synthesis process for $\operatorname{MoS}_{2}$-SILM (f) ${ }^{[69]}$

(b) GO membrane; (c) $\mathrm{MoS}_{2}$ membrane; (d) GO-MoS 2 (50/50) hybrid membrane; (e) GO-MoS 2 (75/25) hybrid membrane

有明显改变。据此, 他们提出 $\mathrm{MoS}_{2}$ 层状膜由少层紧 密堆叠的层片束(Bundler)组成(图 10(a)), 气体分子 的传质路径包括单层之间的小空间(Interlayer space), 和层片束之间的大空间(Inter-bundler space), 晶面
间距(即小空间的层间距)的减小拓宽了层片束之间 的大空间，导致膜的气体渗透率增加。

相比于 GO 层状膜, TMDs 层状膜由于二维通 道的间距更大、缺陷更多, 对 $\mathrm{H}_{2}$ 的选择性较差, 但 
具有渗透率高的优点。Ostwal 等 ${ }^{[64]}$ 利用 GO 更易 组装成膜的特性, 将 $\mathrm{GO}$ 与 $\mathrm{MoS}_{2}$ 复合, 使之组装 得更加紧密与均匀, 从而提高 $\mathrm{MoS}_{2}$ 膜对 $\mathrm{H}_{2} / \mathrm{CO}_{2}$ 的选择性, 同时保持 $\mathrm{MoS}_{2}$ 膜高气体渗透率的特点 (图 10(b e))。

此外, $\mathrm{MoS}_{2}$ 的缺陷位点对 $\mathrm{CO}_{2}$ 具有较强的吸附 作用 ${ }^{[65-68]}$, 这使得更多的研究集中于其在 $\mathrm{CO}_{2}$ 分离 上的应用。Shen 等 ${ }^{[68]}$ 在聚醚嵌段聚酰胺(Pebax)膜 中加入 $0.15 \mathrm{wt} \%$ 的 $\mathrm{MoS}_{2}$, Pebax 膜通过溶解-扩散 机制分离气体, $\mathrm{MoS}_{2}$ 对 $\mathrm{CO}_{2}$ 的吸附作用可以提高 $\mathrm{CO}_{2}$ 的溶解度, 从而提高膜对 $\mathrm{CO}_{2} / \mathrm{N}_{2}$ 的选择性(93) 和 $\mathrm{CO}_{2}$ 渗透率 $\left(2.14 \times 10^{-14} \mathrm{~mol} \cdot \mathrm{m}^{-} \mathrm{m}^{-2} \cdot \mathrm{s}^{-1} \cdot \mathrm{Pa}^{-1}\right)$ 。 Peng 等 ${ }^{[69-70]}$ 先后制备出 $\mathrm{MoS}_{2}-\mathrm{SILMs}^{[69]}$ (图 10(f)) 和 $\mathrm{WS}_{2}-\mathrm{SILMs}^{[70]}$, 对 $\mathrm{CO}_{2}$ 的选择性均远超过纯的 TMDs 膜和[BMIM] $\left[\mathrm{BF}_{4}\right]$ 膜, 然而与 GO-SILMs 相比 仍有一定差距，可能是由于 GO-SILMs 的成分和结 构更均匀，而 TMDs-SILM 的褶皱和缺陷更多。

TMDs 具有良好的机械强度、热稳定性和化学 稳定性, 吸附 $\mathrm{CO}_{2}$ 的特性使之在 $\mathrm{CO}_{2}$ 分离领域有一 定的应用前景。然而, 其原材料的丰度不如碳材料, 膜的分离性能有待进一步提升。开发 TMDs 混合基 质膜，保持其高渗透率的同时提高选择性是目前的 研究方向之一。

\section{4 二维过渡金属碳化物/氮化物}

二维过渡金属碳化物/氮化物(MXene)是一种新 兴的 2D 材料, 通常由 MAX 相陶瓷经插层、刻蚀、 剥离而得 ${ }^{[71]}$, 表面的 $-\mathrm{F},-\mathrm{OH}$ 和 $-\mathrm{O}$ 等官能团使之亲 水，可以配置水溶液，通过真空抽滤、旋涂、喷涂等 方法制备成薄膜。MXene 膜具有诸多优点，例如超 快的水渗透率 ${ }^{[72-73]}$ 、精细的离子篮分性能 ${ }^{[74]}$ 、优异 的抗菌能力 ${ }^{[75-76]}$ 等。近年的研究发现, MXene 膜应 用于气体分离也有突出的效果 ${ }^{[77-78]}$ 。

Wang 课题组 ${ }^{[77]}$ 对 MXene 的应用进行了较多的 研究。他们将真空抽滤获得的 $\mathrm{Ti}_{3} \mathrm{C}_{2} \mathrm{~T}_{x}$ 自支撑层状膜 用于气体篮分, 发现其具有优异的 $\mathrm{H}_{2}$ 渗透率 $\left(>7 \times 10^{-13} \mathrm{~mol} \cdot \mathrm{m}^{\prime} \cdot \mathrm{m}^{-2} \cdot \mathrm{s}^{-1} \cdot \mathrm{Pa}^{-1}\right)$ 和较高的 $\mathrm{H}_{2} / \mathrm{CO}_{2}$ 选择 性 $(>160)$ 。 MXene 膜的层片之间的纳米通道与 GO 膜相似，均是利用尺寸效应分离气体(图 11(a d))。 通过计算 ${ }^{[79]}$, 他们系统研究了多种气体(包括 $\mathrm{He}$ 、 $\mathrm{H}_{2} 、 \mathrm{CO}_{2} 、 \mathrm{~N}_{2}$ 和 $\mathrm{CH}_{4}$ ) 在 2D MXene 纳米通道中的扩 散机制, 发现气体分子的结构因素(如尺寸、质量和 极性等)和 MXene 膜的结构因素(如层间距和是否含 水分子等)均对气体的扩散有显著影响。

Jin 等 ${ }^{[78]}$ 用嗍酸和聚乙烯亚胺(PEI)修饰 MXene, 实现了 MXene 组装性质和层间距的改变。未修饰的
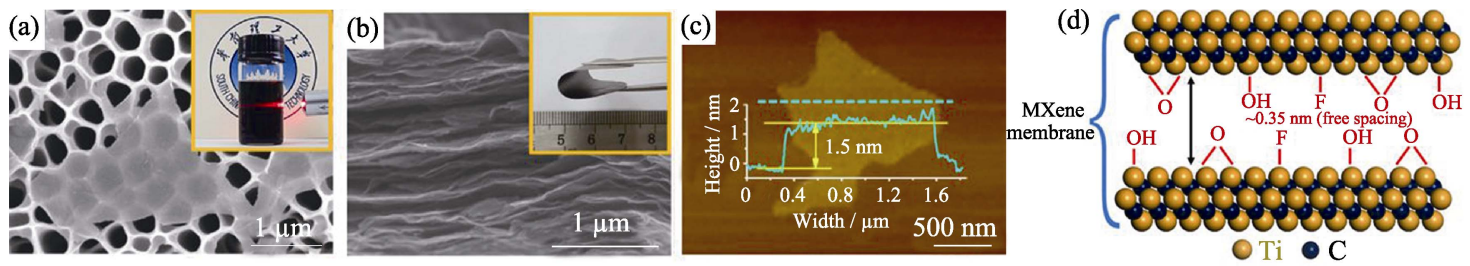

(e)
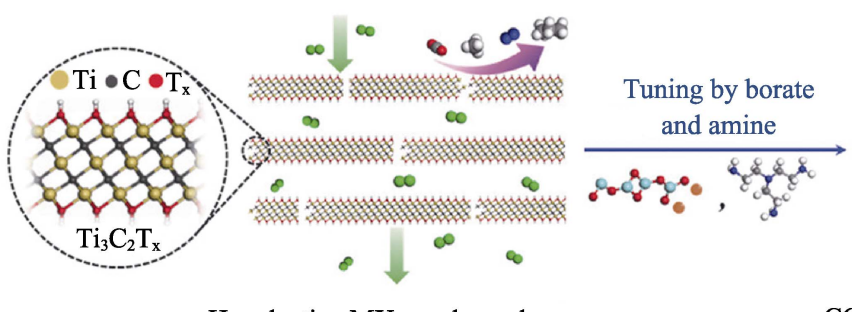

$\mathrm{H}_{2}$-selective MXene channels

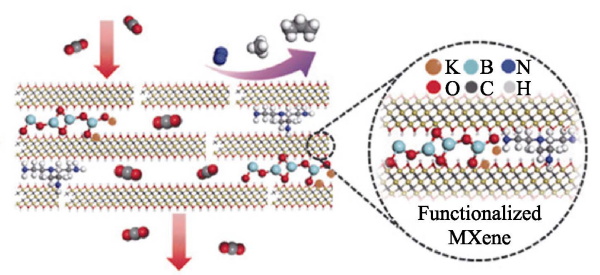

$\mathrm{CO}_{2}$-selective MXene channels

$$
\infty: \mathrm{H}_{2} \infty: \mathrm{CO}_{2} \infty: \mathrm{N}_{2} \oplus: \mathrm{CH}_{4} \oplus: \mathrm{C}_{3} \mathrm{H}_{8}
$$

图 11 AAO 祄底上 MXene 的 SEM 照片(a) (插图为 MXene 胶体水溶液的科根达尔效应), MXene 膜的截面 SEM 照片(b) (插图为弯折的膜), MXene 片层的 AFM 图(c), 相邻 MXene 纳米片示意图(d) ${ }^{[77]}$,

以及 MXene 膜分别选择性渗透 $\mathrm{H}_{2}$ 和 $\mathrm{CO}_{2}$ 的示意图(e) ${ }^{[78]}$

Fig. 11 SEM image of the delaminated MXene nanosheets on porous anodic aluminum oxide (AAO) (a) with insert showing the Tyndall scattering effect in MXene colloidal solution in water, cross-sectional SEM image of the MXene membrane

(b) with insert showing a tweezer bent membrane, AFM image of the Mxene nanosheet on cleaved mica (c), illustration of the spacing between the neighboring MXene nanosheets in the membrane (d) ${ }^{[77]}$, structures and gas transport of $\mathrm{H}_{2}$-selective and $\mathrm{CO}_{2}$-selective Mxene nanofilms $(\mathrm{e})^{[78]}$ 
MXene 膜是扩散控制的渗透机制，基于尺寸效应， 有利于选择渗透 $\mathrm{H}_{2}$ 。而嗍酸和 PEI 有利于吸附 $\mathrm{CO}_{2}$, 与 MXene 交联后, 膜对 $\mathrm{CO}_{2}$ 的溶解度增大, 渗透转 变为由溶解控制, 可以有效地从 $\mathrm{N}_{2} 、 \mathrm{CH}_{4}$ 等气体中 分离 $\mathrm{CO}_{2}$ (图 11(e))。

MXene 一般从 MAX 相中剥离得到, 成本较高, 目前在膜分离领域尤其是气体分离上的报道较少, 其选择性与石墨烯及其衍生物的最优值相比有一定 差距, 但是对 $\mathrm{H}_{2}$ 的渗透率远远大于后者, 机械强度 和化学稳定性好。随着更多类型的高质量 MXene 的开发和膜制备方法的不断优化, MXene 基膜的分 离性能有望进一步提高。

\section{5 其他无机二维材料}

其他无机 $2 \mathrm{D}$ 材料, 如层状双氢氧化物、六方氮 化硼、云母纳米片等, 近年来也在气体分离膜领域 有所研究。

层状双氢氧化物 (LDH) 是一类离子型层状化合 物, 由以金属离子为中心的共边缘八面体单元组成, 中间层为电荷补偿阴离子和溶剂化分子 ${ }^{[80-81]}$ 。 Tsotsis 等 ${ }^{[22-83]}$ 先后用电泳沉积法和真空抽吸法制备 出 $\mathrm{MgAl}-\mathrm{CO}_{3} \mathrm{LDH}$ 薄膜, 具有较低的气体选择性, 大量介孔和针孔的存在使其气体分离性能较差。为 了制备更高质量的 $\mathrm{LDH}$ 薄膜, Caro 等 ${ }^{[84]}$ 采用原位生 长法, 利用 $\gamma-\mathrm{Al}_{2} \mathrm{O}_{3}$ 修饰的 $\alpha-\mathrm{Al}_{2} \mathrm{O}_{3}$ 祄底和 $\mathrm{CO}_{2}$ 饱和 溶液制得高质量的 $\mathrm{NiAl}-\mathrm{CO}_{3}$ 膜, 高度约为 $0.31 \mathrm{~nm}$ 的层间通道起到分子篎的作用, 对 $\mathrm{H}_{2} / \mathrm{CH}_{4}$ 的选择性 约为 80 。
六方氮化嗍纳米片 (h-BN), 被称为“白色石墨 烯”, 是由 $\mathrm{sp}^{2}$ 杂化的 $\mathrm{B}$ 原子和 $\mathrm{N}$ 原子以六边形网络 交替连接构成的 $2 \mathrm{D}$ 材料。Wang 等 ${ }^{[85]}$ 将氨基官能化 的氮化嗍纳米片 $(\mathrm{FBN})$ 结合到交联的热重排的聚合 物(XTR)中, 制备出 FBN-XTR 复合膜, 这是首次在 XTR 中加入热稳定的无机填料。此前, 研究人员在 $\mathrm{XTR}$ 中加入 $\mathrm{GO}$ 作为填料 ${ }^{[86]}$, 但是 GO 在热处理时 被还原, 限制了分离性能的提升。而 h-BN 耐高温的 特性使其在 $425{ }^{\circ} \mathrm{C}$ 热处理后保持不变, 膜的机械强 度和氢选择性大大提高。

近年来, 云母纳米片由于其高介电强度、高化 学稳定性和热稳定性等优点, 被广泛应用于电子领 域 ${ }^{[87-88]}$ 。Peng 课题组 ${ }^{[89]}$ 借鉴离子液体混合基质膜的 思路, 将 IL 填充于云母纳米片层状膜中, 带负电的 云母片可以吸引 IL 中的阳离子, 使之与阴离子分离, 阴离子吸附 $\mathrm{CO}_{2}$ 从而提高膜的选择性。

黑磷作为一种新型 $2 \mathrm{D}$ 材料, 尚未被应用于气 体分离膜领域, 但已有计算开始研究黑磷的气体吸 附性能。Tang 的课题组 ${ }^{[90-91]}$ 通过 DFT 计算研究了 掺杂的黑磷与 $\mathrm{H}_{2}$ 和 $\mathrm{CO}_{2}$ 的相互作用, 为黑磷在气体 吸附和分离领域的应用提供了一定的理论基础。

多种无机 $2 \mathrm{D}$ 材料在气体分离膜领域的尝试应 用为膜的发展带来机遇, 同时也带来诸多挑战。如 LDH 的柔韧性低、表面较石墨烯更为粗糙, 组装的 层状膜缺陷较多, 而 h-BN 等存在表面含氧官能团 较少、与聚合物相容性差、难以进一步改性和功能 化等问题。这些二维材料在气体分离膜领域的研究 刚刚起步, 目前报道较少, 性能尚不如石墨烯膜, 有待进一步研究与提升。表 2 总结了除石墨烯基材

表 2 其他新型无机 2D 材料气体膜分离性能比较

Table 2 Other novel inorganic 2DMs for membrane gas separation

\begin{tabular}{|c|c|c|c|c|c|}
\hline Materials $^{\mathrm{a}}$ & Preparation method & Feed condition & Selectivity & Permeate rate/permeance/ permeability & Ref. \\
\hline $\mathrm{MoS}_{2} / \mathrm{AAO}$ & Vacuum filtration & $\mathrm{H}_{2} / \mathrm{CO}_{2}$ & 3.4 & $9.19 \times 10^{-6} \mathrm{~mol} \cdot \mathrm{m}^{-2} \cdot \mathrm{s}^{-1} \cdot \mathrm{Pa}^{-1}$ & [62] \\
\hline $\mathrm{MoS}_{2}$ & Vacuum filtration & $\mathrm{H}_{2} / \mathrm{CO}_{2}$ & 8.29 & $3.94 \times 10^{-13} \mathrm{~mol} \cdot \mathrm{m} \cdot \mathrm{m}^{-2} \cdot \mathrm{s}^{-1} \cdot \mathrm{Pa}^{-1}$ & [63] \\
\hline $\mathrm{GO} / \mathrm{MoS}_{2}$ & Vacuum filtration & $\mathrm{H}_{2} / \mathrm{CO}_{2}$ & 26.7 & $8.04 \times 10^{-7} \mathrm{~mol} \cdot \mathrm{m}^{-2} \cdot \mathrm{s}^{-1} \cdot \mathrm{Pa}^{-1}$ & [64] \\
\hline $\mathrm{MoS}_{2}-$ Pebax & Spin coating & $\mathrm{CO}_{2} / \mathrm{N}_{2}$ & 93 & $2.14 \times 10^{-14} \mathrm{~mol} \cdot \mathrm{m} \cdot \mathrm{m}^{-2} \cdot \mathrm{s}^{-1} \cdot \mathrm{Pa}^{-1}$ & [68] \\
\hline $\mathrm{MoS}_{2}-[\mathrm{BMIM}]\left[\mathrm{BF}_{4}\right]$ & Vacuum filtration and drop & $\mathrm{CO}_{2} / \mathrm{N}_{2}$ & 131.42 & $1.60 \times 10^{-8} \mathrm{~mol} \cdot \mathrm{m}^{-2} \cdot \mathrm{s}^{-1} \cdot \mathrm{Pa}^{-1}$ & [69] \\
\hline $\mathrm{WS}_{2}-[\mathrm{BMIM}]\left[\mathrm{BF}_{4}\right]$ & $\begin{array}{l}\text { Vacuum filtration and } \\
\text { spin-coating }\end{array}$ & $\mathrm{CO}_{2} / \mathrm{N}_{2}$ & 153.21 & $1.58 \times 10^{-8} \mathrm{~mol} \cdot \mathrm{m}^{-2} \cdot \mathrm{s}^{-1} \cdot \mathrm{Pa}^{-1}$ & [70] \\
\hline $\mathrm{Ti}_{2} \mathrm{C}_{3} \mathrm{~T}_{x}$ & Vacuum filtration & $\mathrm{H}_{2} / \mathrm{CO}_{2}$ & 160 & $7.37 \times 10^{-13} \mathrm{~mol} \cdot \mathrm{m} \cdot \mathrm{m}^{-2} \cdot \mathrm{s}^{-1} \cdot \mathrm{Pa}^{-1}$ & [77] \\
\hline $\mathrm{Ti}_{2} \mathrm{C}_{3} \mathrm{~T}_{x} /$ borate-PEI & $\begin{array}{l}\text { Vacuum filtration and } \\
\text { spin-coating }\end{array}$ & $\mathrm{CO}_{2} / \mathrm{CH}_{4}$ & 15.3 & $1.17 \times 10^{-7} \mathrm{~mol} \cdot \mathrm{m}^{-2} \cdot \mathrm{s}^{-1} \cdot \mathrm{Pa}^{-1}$ & [78] \\
\hline $\mathrm{MgAl}-\mathrm{CO}_{3} \mathrm{LDH}$ & Electrophoretic deposition & $\mathrm{CO}_{2} / \mathrm{N}_{2}$ & 1.53 & $2.34 \times 10^{-7} \mathrm{~mol} \cdot \mathrm{m}^{-2} \cdot \mathrm{s}^{-1} \cdot \mathrm{Pa}^{-1}$ & [83] \\
\hline $\mathrm{MgAl}-\mathrm{CO}_{3} \mathrm{LDH}$ & Vacuum-suction & $\mathrm{CO}_{2} / \mathrm{N}_{2}$ & 34.4 & $5.5 \times 10^{-10} \mathrm{~mol} \cdot \mathrm{m}^{-2} \cdot \mathrm{s}^{-1} \cdot \mathrm{Pa}^{-1}$ & [83] \\
\hline $\mathrm{NiAl}-\mathrm{CO}_{3} \mathrm{LDH}$ & In situ growth & $\mathrm{H}_{2} / \mathrm{CH}_{4}$ & 80 & $4.5 \times 10^{-8} \mathrm{~mol} \cdot \mathrm{m}^{-2} \cdot \mathrm{s}^{-1} \cdot \mathrm{Pa}^{-1}$ & [84] \\
\hline h-BN-XTR & Drop casting & $\mathrm{H}_{2} / \mathrm{CH}_{4}$ & 24.1 & $7.03 \times 10^{-14} \mathrm{~mol} \cdot \mathrm{m} \cdot \mathrm{m}^{-2} \cdot \mathrm{s}^{-1} \cdot \mathrm{Pa}^{-1}$ & [85] \\
\hline mica-[BMIM] $\left[\mathrm{BF}_{4}\right]$ & $\begin{array}{l}\text { Vacuum filtration and } \\
\text { drop-casting }\end{array}$ & $\mathrm{CO}_{2} / \mathrm{N}_{2}$ & 87 & $2.68 \times 10^{-8} \mathrm{~mol} \cdot \mathrm{m}^{-2} \cdot \mathrm{s}^{-1} \cdot \mathrm{Pa}^{-1}$ & [89] \\
\hline
\end{tabular}

a PEI: polyethylenimine; XTR: crosslinked thermally rearranged polymer 
料以外其他新型无机 $2 \mathrm{D}$ 材料在气体分离膜领域的 研究进展。

\section{6 总结与展望}

目前，在工业领域广泛应用的有机高分子材料 气体分离膜面临的 Trade-off 效应、老化和塑化等问 题制约了其性能的发展。开发新型膜材料, 并完成 从实验室到大规模工业化应用的转变, 已成为气体 膜分离技术发展的关键。无机 $2 \mathrm{D}$ 材料膜有望同时 实现高渗透率和高选择性, 且具有耐高温、抗腐蚀 的特点, 极大地促进了高性能膜的发展, 然而其在 研发和应用中仍存在诸多问题和挑战，目前尚未有 实际的工业化应用。无机 $2 \mathrm{D}$ 材料气体分离膜面临 的挑战主要如下:

1) 制备气体分离膜的原材料一一如大尺寸、高 质量的石墨烯、MXene 片层等产率较低、成本高、 耗时耗能。需要开发成本更低、可规模化生产的原 材料制备方法, 并进一步研究控制原材料的尺寸和 官能团等结构，以精细调节膜的微结构和化学性质。

2) 目前无机 2D 材料成膜技术尚不成熟，难以 生产大面积、高质量、可工业化应用的膜。对于 NATM, 如多孔石墨烯，在表面制造出尺寸均一可 控的亚纳米孔仍是技术难题, 同时需要更好地理解 在合成、处理过程中可能出现的缺陷来源, 设计限 制缺陷泄漏的方法。对于层状膜，目前主要的成膜 方法为抽滤、旋涂、溶液浇铸等，在成膜时如何对 层间距进行精确调控和设计是应用的关键。

3）混合基质膜兼具有机基质与无机材料的优 点, 是气体分离膜目前的研究热点。需解决的问题 有, 如何保证无机填料的分散性、插层聚合物的均 匀性、提高 $2 \mathrm{D}$ 材料与聚合物的相容性、控制界面 结构以避免缺陷等。

4) 无机 $2 D$ 材料膜的气体分离机理尚在不断发 展与完善, 可以采用先进的表征技术与仿真计算相 结合，对层间通道、缺陷和官能团等对传质过程的 影响进行研究分析, 进一步建立完善的模型与理论, 以指导高性能膜的合成。

5) 目前对分离膜在高温高压和复杂气体环境 下的长时间测试较少，普遍在较为单一的气体环境 或常温、常压下进行测试，实际应用可能比测试环 境更复杂，需要改进和规范可靠性的评价方法。

\section{参考文献:}

[1] LIU G, JIN W, XU N. Graphene-based membranes. Chemical Society Reviews, 2015, 44(15): 5016-5030.
[2] SHOLL D S, LIVELY R P. Seven chemical separations to change the world. Nature, 2016, 532(7600): 435-437.

[3] LI P, WANG Z, QIAO Z, et al. Recent developments in membranes for efficient hydrogen purification. Journal of Membrane Science, 2015. 495: 130-168.

[4] JEON Y W, LEE D H. Gas membranes for $\mathrm{CO}_{2} / \mathrm{CH}_{4}$ (biogas) separation: a review. Environmental Engineering Science, 2015, 32(2): $71-85$.

[5] DALANE K, DAI Z, MOGSETH G, et al. Potential applications of membrane separation for subsea natural gas processing: a review. Journal of Natural Gas Science and Engineering, 2017, 39: 101-117.

[6] HIMMA N F, WARDANI A K, PRASETYA N, et al. Recent progress and challenges in membrane-based $\mathrm{O}_{2} / \mathrm{N}_{2}$ separation. Reviews in Chemical Engineering, 2019, 35(5): 591-625.

[7] ZHU J, HOU J, ULIANA A, et al. The rapid emergence of two-dimensional nanomaterials for high-performance separation membranes. Journal of Materials Chemistry A, 2018, 6(9): 3773-3792.

[8] GIN D L, NOBLE R D. Designing the next generation of chemical separation membranes. Science, 2011, 332(6030): 674.

[9] QIU T, KUANG C, ZHENG X, et al. On the research and application trends of global gas membrane separation technology-based on analysis of SCI articles and patents in recent 20 years. Chemical Industry \& Engineering Progress, 2016, 35(7): 2299-2308.

[10] YAMPOLSKII Y. Polymeric gas separation membranes. Macromolecules, 2012, 45(8): 3298-3311.

[11] PROZOROVSKA L, KIDAMBI P R. State-of-the-art and future prospects for atomically thin membranes from $2 \mathrm{D}$ materials. $A d-$ vanced Materials, 2018, 30(52): 1801179.

[12] LIU M, GURR P A, FU Q, et al. Two-dimensional nanosheet-based gas separation membranes. Journal of Materials Chemistry A, 2018, 6(46): 23169-23196.

[13] LIU G, JIN W, XU N. Two-dimensional-material membranes: a new family of high-performance separation membranes. Angewandte Chemie International Edition, 2016, 55(43): 13384-13397.

[14] WANG L, BOUTILIER M S H, KIDAMBI P R, et al. Fundamental transport mechanisms, fabrication and potential applications of nanoporous atomically thin membranes. Nature Nanotechnology, 2017, 12: 509

[15] WIJMANS J G, BAKER R W J. The solution-diffusion model: a review. Journal of Membrane Science, 1995, 107: 1-21.

[16] LI C, MECKLER S M, SMITH Z P, et al. Engineered transport in microporous materials and membranes for clean energy technologies. Advanced Materials, 2018, 30(8): 1704953.

[17] SHEN J, LIU G, HUANG K, et al. Membranes with fast and selective gas-transport channels of laminar graphene oxide for efficient $\mathrm{CO}_{2}$ capture. Angewandte Chemie International Edition, 2015, 127(2): 588-592.

[18] [18] DRAHUSHUK L W, WANG L, KOENIG S P, et al. Analysis of time-varying, stochastic gas transport through graphene membranes. ACS Nano, 2016, 10(1): 786-795.

[19] KIM H W, YOON H W, YOON S M, et al. Selective gas transport through few-layered graphene and graphene oxide membranes. Science, 2013, 342(6154): 91-95.

[20] LI H, SONG Z, ZHANG X, et al. Ultrathin, molecular-sieving graphene oxide membranes for selective hydrogen separation. Science, 2013, 342(6154): 95-98.

[21] WANG Z, WANG D, ZHANG S, et al. Interfacial design of mixed matrix membranes for improved gas separation performance. $A d$ vanced Materials, 2016, 28(17): 3399-3405.

[22] ZHU X, TIAN C, DO-THANH C L, et al. Two-dimensional materials as prospective scaffolds for mixed-matrix membrane-based 
$\mathrm{CO}_{2}$ separation. ChemSusChem, 2017, 10(17): 3304-3316.

[23] GEIM A K, NOVOSELOV K S. The rise of graphene. Nature Materials, 2007, 6: 183.

[24] PARTOENS B, PEETERS F. From graphene to graphite: electronic structure around the K point. Phys. Rev. B, 2006, 74(7): 075404.

[25] BUNCH J S, VERBRIDGE S S, ALDEN J S, et al. Impermeable atomic membranes from graphene sheets. Nano Letters, 2008, 8(8): 2458-2462.

[26] BERRY V. Impermeability of graphene and its applications. Carbon, 2013, 62: 1-10.

[27] LEE C, WEI X, KYSAR J W, et al. Measurement of the elastic properties and intrinsic strength of monolayer graphene. Science, 2008, 321(5887): 385-388.

[28] JIANG D E, COOPER V, DAI S. Porous graphene as the ultimate membrane for gas separation. Nano Letters, 2009, 9: 4019-4024.

[29] WANG S, DAI S, JIANG D E. Continuously tunable pore size for gas separation via a bilayer nanoporous graphene membrane. ACS Applied Nano Materials, 2019, 2(1): 379-384.

[30] KOENIG S P, WANG L, PELlEGRINO J, et al. Selective molecular sieving through porous graphene. Nature Nanotechnology, 2012, 7(11): 728-732.

[31] BELL D, LEMME M, STERN L, et al. Precision cutting and patterning of graphene with helium ions. Nanotechnology, 2009, 20: 455301.

[32] CELEBI K, BUCHHEIM J, WYSS R M, et al. Ultimate permeation across atomically thin porous graphene. Science, 2014, 344(6181): 289-292.

[33] HUANG S, DAKHCHOUNE M, LUO W, et al. Single-layer graphene membranes by crack-free transfer for gas mixture separation. Nature Communications, 2018, 9(1): 2632-2632.

[34] FISCHBEIN M D, DRNDIĆ M. Electron beam nanosculpting of suspended graphene sheets. Applied Physics Letters, 2008, 93(11): 113107.

[35] LU N, WANG J, FLORESCA H C, et al. In situ studies on the shrinkage and expansion of graphene nanopores under electron beam irradiation at temperatures in the range of $400-1200{ }^{\circ} \mathrm{C}$. Carbon, 2012, 50(8): 2961-2965.

[36] GARAJ S, HUBBARD W, REINA A, et al. Graphene as a subnanometre trans-electrode membrane. Nature, 2010, 467: 190.

[37] MERCHANT C. DNA translocation through graphene nanopores. Biophysical Journal, 2011, 100(3): 521a.

[38] HUMMERS JR W S, OFFEMAN R E. Preparation of graphitic oxide. Journal of the American Chemical Society, 1958, 80(6): 1339.

[39] DIMIEV A, TOUR J. Mechanism of graphene oxide formation. ACS Nano, 2014, 8(3): 3060-3067.

[40] NAIR R R, WU H A, JAYARAM P N, et al. Unimpeded permeation of water through helium-leak-tight graphene-based membranes. Science, 2012, 335(6067): 442-444.

[41] IBRAHIM A, LIN Y S. Gas permeation and separation properties of large-sheet stacked graphene oxide membranes. Journal of Membrane Science, 2018, 550: 238-245.

[42] YANG J, GONG D, LI G, et al. Self-assembly of thiourea-crosslinked graphene oxide framework membranes toward separation of small molecules. Advanced Materials, 2018, 30(16): 1705775.

[43] ABRAHAM J, VASU K S, WILLIAMS C D, et al. Tunable sieving of ions using graphene oxide membranes. Nature Nanotechnology, 2017, 12(6): 546-550.

[44] CHI C, WANG X, PENG Y, et al. Facile preparation of graphene oxide membranes for gas separation. Chemistry of Materials, 2016, 28(9): 2921-2927.

[45] YANG E, HAM M H, PARK H B, et al. Tunable semi-permeability of graphene-based membranes by adjusting reduction degree of laminar graphene oxide layer. Journal of Membrane Science, 2018, 547: 73-79.

[46] SU Y, KRAVETS V G, WONG S L, et al. Impermeable barrier films and protective coatings based on reduced graphene oxide. Nature Communications, 2014, 5(1): 4843.

[47] SUN P, WANG K, ZHU H. Recent developments in graphene-based membranes: structure, mass-transport mechanism and potential applications. Advanced Materials, 2016, 28(12): 2287-2310.

[48] ZHANG Y, ZHANG S, CHUNG T S. Nanometric graphene oxide framework membranes with enhanced heavy metal removal via nanofiltration. Environmental Science \& Technology, 2015, 49(16): 10235-10242.

[49] BURRESS J W, GADIPELLI S, FORD J, et al. Graphene oxide framework materials: theoretical predictions and experimental results. Angewandte Chemie International Edition, 2010, 49(47): 8902-8904.

[50] KARUNAKARAN M, VILLALOBOS L F, KUMAR M, et al. Graphene oxide doped ionic liquid ultrathin composite membranes for efficient $\mathrm{CO}_{2}$ capture. Journal of Materials Chemistry A, 2017, 5(2): 649-656.

[51] WANG S, XIE Y, HE G, et al. Graphene oxide membranes with heterogeneous nanodomains for efficient $\mathrm{CO}_{2}$ separations. Angewandte Chemie, 2017, 129(45): 14434-14439.

[52] HUANG G, ISFAHANI A P, MUCHTAR A, et al. Pebax/ionic liquid modified graphene oxide mixed matrix membranes for enhanced $\mathrm{CO}_{2}$ capture. Journal of Membrane Science, 2018, 565: 370-379.

[53] XIN Q, MA F, ZHANG L, et al. Interface engineering of mixed matrix membrane via $\mathrm{CO}_{2}$-philic polymer brush functionalized graphene oxide nanosheets for efficient gas separation. Journal of Membrane Science, 2019, 586: 23-33.

[54] SHEN J, LIU G, HUANG K, et al. Subnanometer two-dimensional graphene oxide channels for ultrafast gas sieving. ACS Nano, 2016, 10(3): 3398-3409.

[55] YING W, CAI J, ZHOU K, et al. Ionic liquid selectively facilitates $\mathrm{CO}_{2}$ transport through graphene oxide membrane. ACS Nano, 2018, 12(6): 5385-5393.

[56] SCHMIDT H, WANG S, CHU L, et al. Transport properties of monolayer $\mathrm{MoS}_{2}$ grown by chemical vapor deposition. Nano Letters, 2014, 14(4): 1909-1913.

[57] NICOLOSI V, CHHOWALLA M, G KANATZIDIS M, et al. Liquid exfoliation of layered materials. Science, 2013, 340: 1226419.

[58] ZHANG W, HUANG J K, CHEN C H, et al. High-gain phototransistors based on a CVD $\mathrm{MoS}_{2}$ monolayer. Advanced Materials, 2013, 25(25): 3456-3461.

[59] WANG H, FENG H, LI J. Graphene and graphene-like layered transition metal dichalcogenides in energy conversion and storage. Small, 2014, 10(11): 2165-2181.

[60] ZENG Z, SUN T, ZHU J, et al. An effective method for the fabrication of few-layer-thick inorganic nanosheets. Angewandte Chemie International Edition, 2012, 124(36): 9186-9190.

[61] GEE M A, FRINDT R F, JOENSEN P, et al. Inclusion compounds of $\mathrm{MoS}_{2}$. Materials Research Bulletin, 1986, 21(5): 543-549.

[62] WANG D, WANG Z, WANG L, et al. Ultrathin membranes of singlelayered $\mathrm{MoS}_{2}$ nanosheets for high-permeance hydrogen separation. Nanoscale, 2015, 7(42): 17649-17652.

[63] ACHARI A, SAHANA S, ESWARAMOORTHY M. High performance $\mathrm{MoS}_{2}$ membranes: effects of thermally driven phase transition on $\mathrm{CO}_{2}$ separation efficiency. Energy \& Environmental Science, 2016, 9(4): 1224-1228.

[64] OSTWAL M, SHINDE D B, WANG X, et al. Graphene oxidemolybdenum disulfide hybrid membranes for hydrogen separation. Journal of Membrane Science, 2018, 550: 145-154. 
[65] ZHAO S, XUE J, KANG W. Gas adsorption on $\mathrm{MoS}_{2}$ monolayer from first-principles calculations. Chemical Physics Letters, 2014, 595-596: 35-42.

[66] HE Q, ZENG Z, YIN Z, et al. Fabrication of flexible $\mathrm{MoS}_{2}$ thin-film transistor arrays for practical gas-sensing applications. Small, 2012, 8(19): 2994-2999.

[67] BEREAN K J, OU J Z, DAENEKE T, et al. 2D MoS 2 pdms nanocomposites for $\mathrm{NO}_{2}$ separation. Small, 2015, 11(38): 5035-5040.

[68] SHEN Y, WANG H, ZHANG X, et al. $\mathrm{MoS}_{2}$ nanosheets functionalized composite mixed matrix membrane for enhanced $\mathrm{CO}_{2}$ capture via surface drop-coating method. ACS Applied Materials \& Interfaces, 2016, 8(35): 23371-23378.

[69] CHEN D K, YING W, GUO Y, et al. Enhanced gas separation through nanoconfined ionic liquid in laminated $\mathrm{MoS}_{2}$ membrane. ACS Applied Materials \& Interfaces, 2017, 9(50): 44251-44257.

[70] CHEN D, WANG W, YING W, et al. $\mathrm{CO}_{2}$-philic $\mathrm{WS}_{2}$ laminated membranes with a nanoconfined ionic liquid. Journal of Materials Chemistry A, 2018, 6(34): 16566-16573.

[71] ALHABEB M, MALESKI K, ANASORI B, et al. Guidelines for synthesis and processing of two-dimensional titanium carbide ( $\mathrm{Ti}_{3} \mathrm{C}_{2} \mathrm{~T}_{x}$ MXene). Chemistry of Materials, 2017, 29(18): 7633-7644.

[72] WANG J T, CHEN P P, SHI B B, et al. A regularly channeled lamellar membrane for unparalleled water and organics permeation. Angewandte Chemie-International Edition, 2018, 57(23): 6814-6818.

[73] DING L, WEI Y, WANG Y, et al. A two-dimensional lamellar membrane: MXene nanosheet stacks. Angewandte Chemie International Edition, 2017, 56(7): 1825-1829.

[74] REN C E, HATZELL K B, ALHABEB M, et al. Charge- and size-selective ion sieving through $\mathrm{Ti}_{3} \mathrm{C}_{2} \mathrm{~T}_{x}$ MXene membranes. The Journal of Physical Chemistry Letters, 2015, 6(20): 4026-4031.

[75] RASOOL K, MAHMOUD K A, JOHNSON D J, et al. Efficient antibacterial membrane based on two-dimensional $\mathrm{Ti}_{3} \mathrm{C}_{2} \mathrm{~T}_{x}$ (MXene) nanosheets. Sci. Rep, 2017, 7(1): 1598.

[76] RASOOL K, HELAL M, ALI A, et al. Antibacterial activity of $\mathrm{Ti}_{3} \mathrm{C}_{2} \mathrm{~T}_{x}$ MXene. ACS Nano, 2016, 10(3): 3674-3684.

[77] DING L, WEI Y, LI L, et al. MXene molecular sieving membranes for highly efficient gas separation. Nature Communications, 2018, 9: 155 .

[78] SHEN J, LIU G Z, JI Y F, et al. 2D MXene nanofilms with tunable gas transport channels. Advanced Functional Materials, 2018, 28(31): 1801511 .

[79] LI L, ZHANG T, DUAN Y, et al. Selective gas diffusion in two-dimensional MXene lamellar membranes: insights from molecular dynamics simulations. Journal of Materials Chemistry A, 2018, 6(25): 11734-11742.

[80] WANG Q, O'HARE D. Recent advances in the synthesis and application of layered double hydroxide (LDH) nanosheets. Chemical Reviews, 2012, 112(7): 4124-4155.

[81] LU P, LIU Y, ZHOU T, et al. Recent advances in layered double hydroxides (LDHs) as two-dimensional membrane materials for gas and liquid separations. Journal of Membrane Science, 2018, 567: 89-103.

[82] KIM T W, SAHIMI M, TSOTSIS T T. Preparation of hydrotalcite thin films using an electrophoretic technique. Industrial \& Engineering Chemistry Research, 2008, 47(23): 9127-9132.

[83] KIM T W, SAHIMI M, TSOTSIS T T. The preparation and characterization of hydrotalcite thin films. Industrial \& Engineering Chemistry Research, 2009, 48(12): 5794-5801.

[84] LIU Y, WANG N, CAO Z, et al. Molecular sieving through interlayer galleries. Journal of Materials Chemistry A, 2014, 2(5): $1235-1238$.

[85] WANG Y, LOW Z X, KIM S, et al. Functionalized boron nitride nanosheets: a thermally rearranged polymer nanocomposite membrane for hydrogen separation. Angewandte Chemie International Edition, 2018, 57(49): 16056-16061.

[86] KIM S, HOU J, WANG Y, et al. Highly permeable thermally rearranged polymer composite membranes with a graphene oxide scaffold for gas separation. Journal of Materials Chemistry A, 2018, 6(17): 7668-7674.

[87] ZHANG X, HE Y, LI R, et al. 2D mica crystal as electret in organic field-effect transistors for multistate memory. Advanced Materials, 2016, 28(19): 3755-3760.

[88] WANG D, YUAN G, HAO G, et al. All-inorganic flexible piezoelectric energy harvester enabled by two-dimensional mica. Nano Energy, 2018, 43: 351-358.

[89] YING W, HAN B, LIN H, et al. Laminated mica nanosheets supported ionic liquid membrane for $\mathrm{CO}_{2}$ separation. Nanotechnology, 2019, 30(38): 385705.

[90] ZHANG H P, HU W, DU A, et al. Doped phosphorene for hydrogen capture: a DFT study. Applied Surface Science, 2018, 433: 249-255.

[91] ZHANG H P, DU A, SHI Q B, et al. Adsorption behavior of $\mathrm{CO}_{2}$ on pristine and doped phosphorenes: a dispersion corrected DFT study. Journal of $\mathrm{CO}_{2}$ Utilization, 2018, 24: 463-470. 